\title{
NEUROPSYCHOPHARMACOLOGY REVIEWS Sleep disturbance in PTSD and other anxiety-related disorders: an updated review of clinical features, physiological characteristics, and psychological and neurobiological mechanisms
}

\author{
This article has been corrected since Advance Online Publication and a correction is also printed in this issue \\ Anne Richards ${ }^{1,2}$, Jennifer C. Kanady ${ }^{1,2}$ and Thomas C. Neylan ${ }^{1,2}$
}

The current report provides an updated review of sleep disturbance in posttraumatic stress disorder and anxiety-related disorders. First, this review provides a summary description of the unique and overlapping clinical characteristics and physiological features of sleep disturbance in specific DSM anxiety-related disorders. Second, this review presents evidence of a bidirectional relationship between sleep disturbance and anxiety-related disorders, and provides a model to explain this relationship by integrating research on psychological and neurocognitive processes with a current understanding of neurobiological pathways. A heuristic neurobiological framework for understanding the bidirectional relationship between abnormalities in sleep and anxiety-related brain pathways is presented. Directions for future research are suggested.

Neuropsychopharmacology (2020) 45:55-73; https://doi.org/10.1038/s41386-019-0486-5

\section{INTRODUCTION}

Sleep is a vital physiological process, disruptions of which affect performance in multiple domains of functioning [1], including but not restricted to cognitive [2,3], emotional [4], metabolic [5], and immunologic [6]. Focusing on posttraumatic stress disorder (PTSD) and other anxiety-related disorders specifically, published research points to a bidirectional relationship between disturbances in sleep and anxiety-related disorders. Neurobiological research ranging from animal research to human neuroimaging and polysomnography-based research, alongside clinical treatment research, provide insights about this relationship, while also highlighting questions that remain to be answered. A heuristic neurobiological framework for this relationship is proposed to contribute to ongoing research in this area.

The study of sleep-wake regulation and the neurobiology of sleep disturbance in humans: an introductory overview

Both human and basic animal research have driven a surge in our understanding of sleep neurobiology in recent decades. In the 1950's and 1960's, researchers coined the terms REM and NREM sleep (REMS, NREMS) after observing that humans cycled through periods of sleep with unique electroencephalography (EEG) signatures combined with distinct eye movement patterns and muscle activity levels [7-9]. Since then, the field of sleep research has started to unravel the significance of sleep and its different visually scored stages (NREM N1, N2, N3, and REMS) for various essential functions, including but not limited to synaptic homeostasis [10, 11], cognitive function [2, 12], emotion regulation [4], memory processing and consolidation [10, 13-15], glucose metabolism [5], immunity [6], and more [1]. Since the mid-20th century as well, numerous studies have also established that circadian and homeostatic processes regulate sleep in humans $[16,17]$. Although understanding the functional neuroanatomy of sleep regulation in humans is still impeded by the challenges of imaging the human brain during sleep, animal research has greatly advanced our grasp of sleep/wake neurocircuitry in mammals, and in combination with pharmacological, EEG, and imaging studies in humans, provides clues regarding the relevance of different brain nuclei and circuits in human sleep.

The existing research points to distinct nuclei and brain regions with prominent and preferential roles in promoting NREMS (which includes slow-wave sleep (SWS/N3 sleep), REMS, and the wake state. Although the most-recent evidence challenges the relative simplicity of sleep/wake neurocircuitry models developed in recent decades (e.g., see [18]), the extant research strongly indicates that specific brain regions and cell types are preferentially active in NREMS, REMS, and/or the wake state, and that these nuclei and cell types influence each other via positive and negative feedback mechanisms in the service of regulating sleep and wake. Figure 1 depicts a current understanding of important brain nuclei involved in NREMS, REMS, and wake regulation. Mutually inhibitory network interactions between NREMS promoting neurons in the ventrolateral preoptic area (VLPO in rodent model; intermediate nucleus (IN) in humans) and wakepromoting neurons in the brainstem and hypothalamus regulate sleep-wake rhythms in a fashion analogous to the engineering concept of a flip-flop switch [19-23]. This model was recently updated to emphasize the importance of fast neurotransmitters, glutamate and GABA [24]. In addition to the VLPO, GABAergic neurons in the medullary parafacial zone (PZ) are also critical for

\footnotetext{
${ }^{1}$ The San Francisco VA Health Care System, San Francisco, CA, USA and ${ }^{2}$ The University of California, San Francisco, San Francisco, CA, USA

Correspondence: Anne Richards (Anne.richards@ucsf.edu)

These authors contributed equally: Dr. Richards, Dr. Kanady
}

Received: 17 March 2019 Revised: 9 August 2019 Accepted: 12 August 2019

Published online: 23 August 2019 


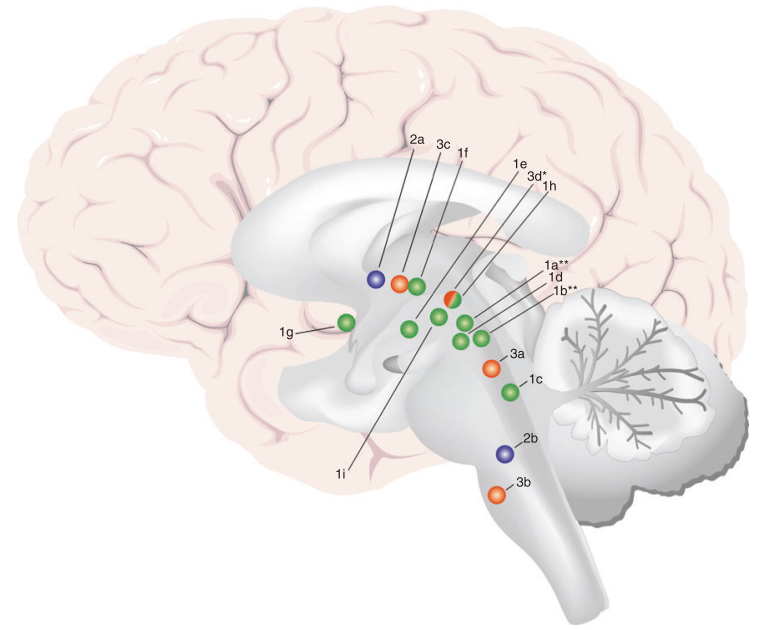

\begin{tabular}{|c|c|c|c|}
\hline $\begin{array}{c}\text { Neuromodulatory } \\
\text { Milieu }\end{array}$ & WAKE & NREMS & REMS \\
\hline Norepinephrine (NE) & $\uparrow$ & $\downarrow$ & $\downarrow$ \\
\hline Serotonin $(5 \mathrm{HT})$ & $\boldsymbol{\top}$ & $\downarrow$ & $\downarrow$ \\
\hline Histamine (Hist) & & $\downarrow$ & $\downarrow$ \\
\hline Acetylcholine (Ach) & $\hat{4}$ & $\downarrow$ & $\uparrow$ \\
\hline Hypocretin/Orexin (Hct) & $\boldsymbol{4}$ & $\downarrow$ & $\downarrow$ \\
\hline Glutamate (Glu) & $\uparrow$ & $\downarrow$ & $\uparrow$ \\
\hline \multicolumn{4}{|c|}{$\begin{array}{l}\text { Above figure and table adaped from Saper papers (refrence } 27 \text { and 24) Nir and Tononi (2010). } \\
\text { Dreaming and the Brain: From Phenomenology to Neurophysiology. Trends in Cognitive Science. } \\
\text { Dark green=highly active; light green=active; pink=reduced; fuscia=extremely reduced or absent. }\end{array}$} \\
\hline
\end{tabular}

Fig. 1 Important wake, NREM sleep, and REM sleep-regulating brain structures and/or nuclei, and associated neuromodulatory milieus. Wakepromoting regions, in green 1a-i; NREMS (including SWS) promoting regions in blue $\mathbf{2 a}-\mathbf{b}$; REM sleep-regulating regions in orange (3a-d). Wake (green): 1a: lateraldorsal tegmentum (LDT; Ach); 1b: pedunculopontine tegmentum (PPT; Ach); 1c: locus coeruleus (LC; NE); 1d: dorsal raphe nucleus (DRN; $5 \mathrm{HT}$ ); 1e: tuberomammillary nucleus (TMN; Hist); 1f: hypocretin neurons of Lateral Hypothalamus (LH; Hct); 1g: cholinergic neurons of basal forebrain (BF; Ach); $1 \mathbf{h}$ : ventrolateral periaqueductal gray (vIPAG; DA); 1i: ventral tegmental area (VTA, DA). NREMS (blue): 2a: ventrolateral preoptic nucleus (VLPO; GABA, galanin; human homolog: intermediate nucleus (IN)); 2b: parafacial zone (PZ; GABA). REMS (orange): 3a: sublaterodorsal tegmental nucleus (SDT; Glu); 3b: ventral gigantocellular reticular nuclei (mediate REM-related muscle atonia; GABA/glycine); 3c: MCH neurons of lateral hypothalamus (LH; MCH/GABA); 3d: ventrolateral periaqueductal gray (vIPAG; GABA), * which is REM-suppressing. **LDT and PPT Ach neurons are also active during and may promote REMS, but may not play a central regulatory role

slow-wave sleep (SWS) homeostasis, shown by anatomic, electrophysiologic, chemogenetic and optogenetic studies in mice $[25,26]$. For example, lesions in GABAergic neurons in $P Z$ increased wakefulness by $50 \%$ and severely disrupted sleep, including a marked reduction of SWS [25]. Brain regions specifically or preferentially active during REMS have also been identified (Fig. 1). Of critical importance in the context of a discussion of anxiety disorders is that arousal/wake centers also broadly innervate the cerebral cortex, and the cerebral cortex reciprocally innervates arousal centers $[24,27]$. This means that in the context of salient information to the cortex, for example from the amygdala, arousal may be elicited despite competing sleeppromoting signals from sleep-specific centers of the brain.

With respect to the study of sleep in psychiatric disorders, scalpEEG-based measurement of cortical physiology remains the mostcommonly utilized tool for studying the neurophysiology of sleep disturbance. Some researchers have also successfully probed the deeper functional neuroanatomy of human sleep disturbance in anxiety-related disorders utilizing neuroimaging techniques [2830], but such studies are still relatively few. Figure 2 depicts the polysomnographic characteristics associated with different stages of sleep, along with a list of their associated clinical features and proposed or evidence-based functions, selected for their pertinence to anxiety disorders. Anxiety-disorder-related findings that will be discussed in greater detail in the following sections include reductions in sleep duration and sleep continuity, reductions in SWS, increases in lighter stages of sleep (NREM N1 and/or N2), and a variety of abnormalities in REMS, including disrupted REMS in anxiety subjects relative to controls. Based on the proposed functions of these various stages of sleep, one can infer numerous neurocognitive and psychological consequences relevant to anxiety, including disturbances in cognitive control, emotion regulation, memory consolidation, and emotional memory processing.

Neurobiology of anxiety-related disorders: a brief overview of neurocircuitry

An overview of the neurobiology of anxiety-related disorders must begin with a definition of these disorders. Given the everchanging landscape of DSM-defined anxiety disorders, and given that all mental disorders may contain or produce a pronounced anxiety component (take paranoid schizophrenia, for example, in which patients may experience core psychotic symptoms resulting in profound and debilitating anxiety), we refer here to anxietyrelated disorders as disorders in which fear and/or anxiety are considered primary and core features of the disorder. As such, we limit our detailed review of sleep disturbance to PTSD, Generalized Anxiety Disorder (GAD), Panic Disorder (PD), Social Phobia (SP), and Specific Phobias (SP). We also include obsessive-compulsive disorder (OCD) owing to its recent (DSM-IV) categorization as an anxiety disorder, because there are significant anxiety components to this disorder, and because we believe this will be of interest to readers.

With these definitions in mind, neuroimaging research in humans has provided relatively greater insight (relative to sleep disturbance in humans) into the functional neuroanatomy and neurocircuitry of anxiety-related disorders [31-35]. Figure 3 depicts the main brain regions broadly implicated in anxietyrelated disorders. The amygdala and insula, key structures involved in the processing of aversive stimuli and in the expression of fear and anxiety, most consistently display hyperactivation in (aversive) task-based neuroimaging studies of anxiety-related disorders (see Duval et al., [34] for a review including disorders of interest minus $O C D$ ). In contrast, the medial prefrontal cortex (mPFC) tends to display hypoactivation in anxiety-related disorders, especially PTSD and GAD, and treatment of anxiety-related disorders has been associated with increases in mPFC activation that relate to symptom improvement [34]. In addition, the dorsal anterior cingulate cortex (dACC), with some exceptions, shows increased activation in anxiety-related disorders, which is thought to reflect the role of dACC in processing and expression of fear and anxiety. In contrast, the rostral ACC ( $\mathrm{ACC})$ is proposed to have a fear-modulating role based on neuroimaging findings. Functional connectivity studies in anxietyrelated disorders generally indicate decreased connectivity between regions that modulate negative emotions (mPFC/rACC) and emotion-processing regions (amygdala, insula).

Other regions are considered to be relevant to anxiety-related disorders, some of which are less well understood or are associated with less consistent or broadly generalizable findings 

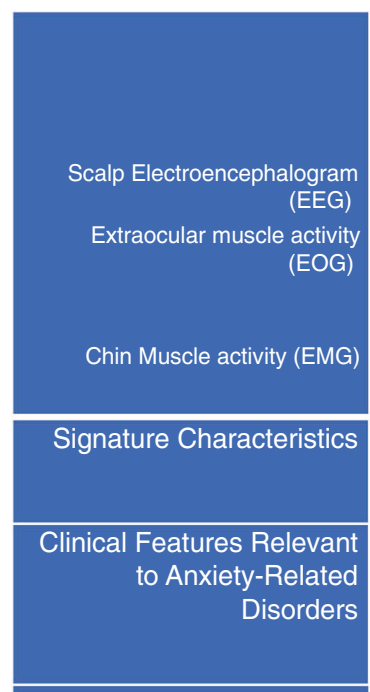

Physiological Functions Relevant to AnxietyRelated Disorders

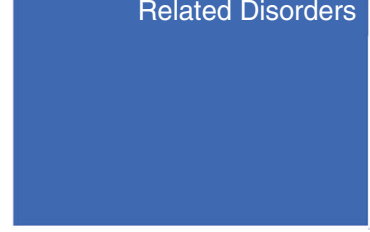

\section{NREM N2}

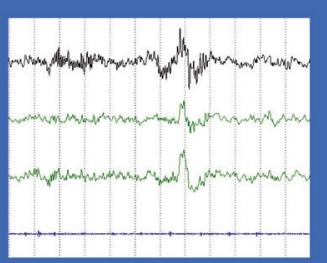

Attenuation and slowing of EEG signal relative to wake; slow rolling eye movements

- Nightmares (PTSD)

- Nightmare Enactment? (PTSD)
Sleep Spindles (brief 11-16 Hz

bursts)

$\mathrm{K}$ complexes (slow, high

amplitude)

- Nightmares (PTSD)

- Nightmare Enactment?

(PTSD)

- Nocturnal Panic Attacks and

Fearful Awakenings

(PD,PTSD)

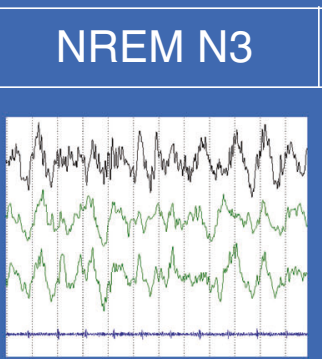

Low frequency $(1-4 \mathrm{~Hz})$

slow waves

(

- Nocturnal Panic Attacks and
Fearful Awakenings (PD,

PTSD)

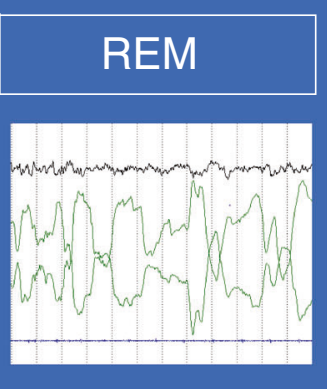

Mixed amplitude, mixed frequency EEG; pronounced eye movements during phasic REMS; flat chin EMG - Nightmares (PTSD) - Nightmare Enactment (PTSD)

- Fearful Awakenings (PTSD)

- Apnea/Hypopnea more frequent in REMS

SLEEP OVERALL: Sufficient sleep duration is essential for cognitive function, including executive functions such as inhibitory control and emotion regulation. Sleep is considered essential for memory consolidation "off-line." Insufficient sleep is associated with HPA axis dysregulation and immune dysfunction/inflammation.

\section{NREM SLEEP:}

N1: very light sleep: transitional between wake and sleep; specific benefits/functions unknown
N2: Spindles implicated in memory consolidation processes; $\mathrm{K}$ complexes and spindles implicated in sleepprotective sensory gating during sleep

\section{REM SLEEP:}

N3: Large slow waves reflect broadly synchronized activity of cortical neurons, considered essential for broad homeostatic recovery, including synaptic homeostatic functions relevant to memory/cognition
Emotional Processing Function proposed

Emotional/Fear Learning and

Memory (i.e. extinction learning, safety learning)

Fig. 2 Polysomnographic characteristics of different sleep stages in humans, along with a list of their associated clinical features and proposed or evidence-based functions selected for their pertinence to anxiety disorders. Some studies have studied the effects of sleep overall (especially sleep duration) on performance in various domains, such as executive cognitive function and emotion regulation, whereas other studies have examined the role of specific sleep stages on performance of various functions more specifically

[34]. For example, the hippocampus is considered to play an emotion-modulating role in fear and anxiety, and often shows hyperactivation in imaging studies of anxiety-related disorders. The dorsolateral prefrontal cortex (dIPFC), implicated in emotion modulation and attention control, has shown patterns of hyperand hypoactivation in anxiety-related disorders. Finally, the thalamus has been shown to display excess activation in taskbased neuroimaging studies in PTSD, Social Anxiety Disorder $(S A D)$, and SD relative to controls. The thalamus is also a key structure involved in the generation of SWS as well as the downregulation of sensory processing during sleep.

Anxiety and sleep circuitry: partially overlapping,

intercommunicating neurocircuits

Although we describe two distinct sets of brain regions implicated in sleep/wake regulation and anxiety states, there is clear evidence of interaction between them, the extent and details of which are only beginning to be uncovered. There are obvious ways in which these communicate, including the aforementioned tight connection between the arousal centers and the cerebral cortex. This shared broad connectivity with the cerebral cortex provides a concrete neurobiological pathway for interconnectivity, but also provides an anatomical context for the cognitive hyperarousal that may link anxiety to sleep disturbance. In addition, the locus coeruleus, the main generator of norepinephrine in the central nervous system, plays both an important role in sleep/wake regulation as well as in anxiety-related processes through its inputs to and from the amygdala as well as the cerebral cortex. We will return to these neurobiological pathways later. A closer look at the clinical and sleep-physiological findings pertaining to the disorders of interest will create a phenomenological background for a subsequent discussion of these neurobiological pathways.

\section{SLEEP DISTURBANCE IN ANXIETY-RELATED DISORDERS: A REVIEW OF CLINICAL FEATURES}

The term "sleep disturbance" in published research on sleep and anxiety-related disorders refers to a range of subjective complaints, sleep disorder diagnoses, and alterations in subjective and objective measures of sleep when clinical samples are compared with controls. Several types of clinical sleep disturbance stand out in this literature; including DSM insomnia disorder diagnoses or symptoms per se, more broadly defined disturbances in subjective sleep quality as measured using various self-report surveys, nightmares, fearful awakenings, nocturnal panic attacks, obstructive sleep apnea (OSA), and abnormal movements during sleep ranging from periodic limb movements to dream enactment behaviors. Certain types of sleep disturbance are more common across anxiety-related disorders (e.g., insomnia and/or broadly defined subjective sleep quality disturbances [36-40] than others (e.g., nightmares) and individuals with anxiety-related disorders often experience more than one type of sleep disturbance. A recent comprehensive publication by Cox and Olatunji provides a highly informative review of sleep disturbance in anxiety-related disorders and the clinical features are given relatively brief review here, where we focus on elements that are less emphasized in that review [41].

\section{Clinical features of sleep disturbance: PTSD}

Subjective sleep disturbance is highly comorbid with PTSD and is often considered a "hallmark" feature of the disorder [42, 43]. Subjective sleep disturbance and recurrent nightmares are listed as diagnostic criteria with prevalence rates as high as $90 \%[36,44-$ 46]. It is therefore rare to encounter a patient with clinically significant non-sleep symptoms of PTSD who does not also report non-restorative and/or disrupted sleep, or distressing dreams. Sleep disturbance in PTSD may also be the most well-studied as 

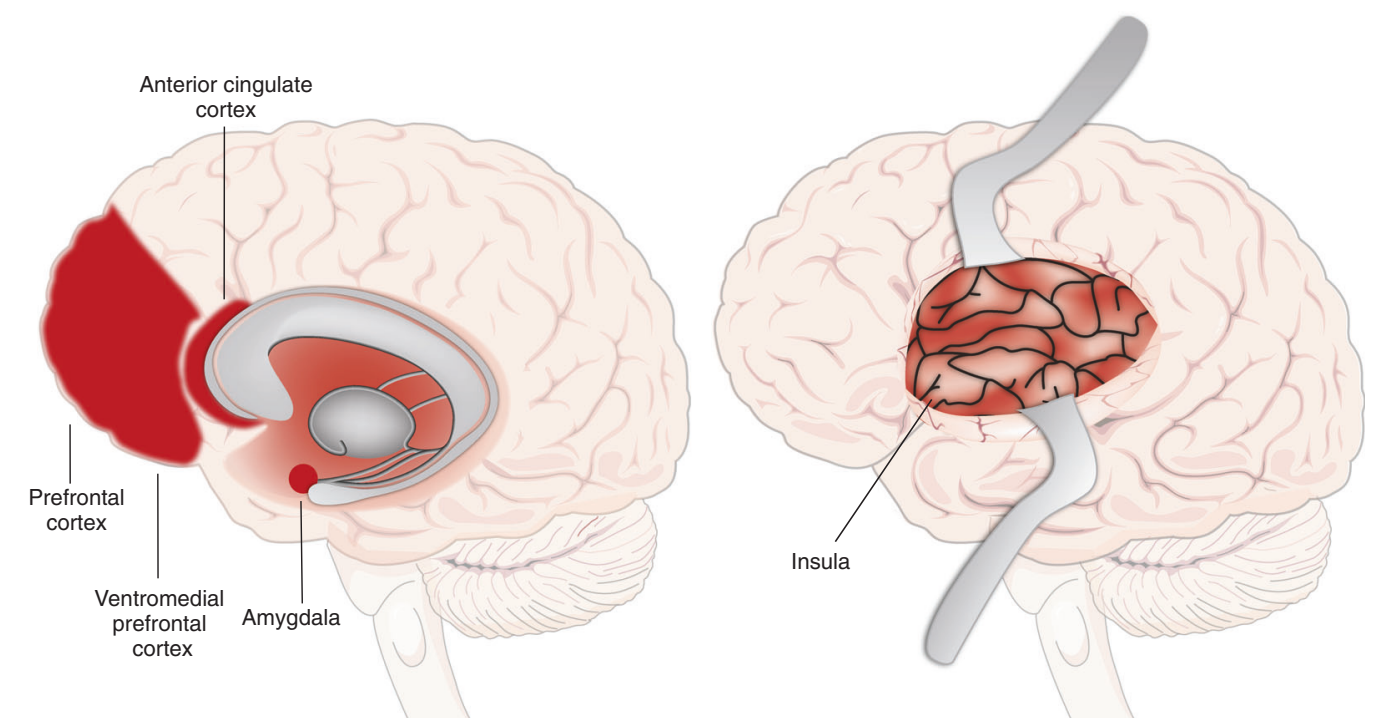

Fig. 3 Important brain regions involved in fear, threat, and anxiety expression and modulation. Neuroimaging research indicates that the amygdala and insula are involved in the expression of fear, threat and anxiety. The dorsal anterior cingulate cortex (dACC) is more involved in the processing and expression of anxiety and fear, whereas the rostral ACC (rACC) is more involved in their modulation. The medial prefrontal cortex (mPFC) is involved in their modulation, especially the ventromedial PFC (vmPFC)

compared with sleep disturbance in other anxiety-related disorders, and therefore may provide the best clues for understanding the sleep-anxiety relationship [41].

Although comorbid insomnia symptoms and poor subjective sleep quality are pervasive features of PTSD, nightmares stand out as a particularly unique feature of this disorder. Prevalence rates for nightmares vary widely, ranging from 19 to $96 \%$ [39, 47, 48], however ample research indicates that the prevalence of nightmares is significantly higher in individuals with PTSD than the general population $[45,49]$ and other psychiatric conditions $[47,48,50,51]$. The wide range is likely attributable to differences in data collection methods, differences in study sample characteristics, and differences in trauma nightmare definitions. For example, most research uses retrospective measures, which are likely to underestimate nightmare frequency compared with prospective log assessments [52]. In addition, the definition of trauma nightmares has changed with various iterations of the DSM and varies widely across studies. Research has demonstrated higher rates of trauma nightmares in females compared with males $[53,54]$ and nightmares may be more frequent in the context of recent trauma compared with chronic PTSD [55].

In addition to the highly prevalent disturbances of insomnia and nightmares, it is now well-established that other sleep disturbances also occur following trauma and/or in PTSD [56-60]. For example, several studies have indicated that panicked awakenings from sleep with poor or no recall of dream content are common in PTSD [59]. Several studies have also reported increases in physical movement during sleep when compared with controls, including periodic leg movements [59] (although see Woodward et al. [61] reporting decreased movement in PTSD sleep). Nightmare enactment is also commonly reported in clinical practice, although prevalence rates are poorly established. Nightmare enactment has typically been observed in younger individuals and is more common immediately following trauma. However, most studies have been conducted in combat veterans, for whom age and time since trauma are difficult to disentangle (e.g., military personnel are exposed at a young age), and thus warrants study in other populations.

Based on the unique combination of sleep symptoms following trauma, Mysliwiec et al. [57, 62] have proposed a new disorder, "trauma-associated sleep disorder". The proposed disorder encompasses disruptive nocturnal behaviors such as thrashing movements panic-like, or startled awakenings, and dream enactment that are proposed to occur out of both REM and NREM sleep. Further research is necessary to determine whether these sleep symptoms represent an early and severe form of sleep response to trauma that later evolves into the more typically described sleep presentation (e.g., insomnia and nightmares), or whether this trajectory is distinct, and possibly even related to neurodegenerative risk. For example, dream enactment is welldescribed in REM Behavior Disorder (RBD) and is considered a prodromal marker of Parkinson's Disease. It remains to be seen whether dream enactment following trauma is unique or is related to the dream enactment characteristic of RBD.

Clinical features of sleep disturbance: GAD

GAD is the other anxiety-related disorder whose DSM diagnostic criteria specifically include sleep disturbance [46]. Despite the inclusion of sleep disturbance in the nosological definition, empirical investigations of sleep disturbance in GAD are relatively limited. Nonetheless, existing published research fairly consistently indicates that subjects with GAD report worse subjective sleep than healthy controls [41]. Sleep disturbance has been reported in up to $75 \%$ of individuals with $\operatorname{GAD}[63,64]$. Adults with GAD report poorer subjective sleep quality [65-67] and have been found to be more likely to have a sleep disorder (vaguely defined, as per chart review) when compared with control groups [68].

Clinical features of sleep disturbance: PD

The extant research has demonstrated that subjective sleep complaints are common in PD, with controlled studies indicating greater difficulties with sleep initiation, sleep maintenance, early morning awakenings and more general sleep quality disturbances in PD subjects than healthy controls [41, 69-73].

A PD-specific sleep disturbance that may overlap with panicked awakenings seen in PTSD are nocturnal panic attacks. Nocturnal panic attacks are characterized by abrupt awakenings from sleep with panic attack symptoms [74]. These can be distinguished from sleep terrors, which are a parasomnia involving severe autonomic arousal and fearful behavior during NREM sleep, and which are generally not recalled subsequently [75]. Although epidemiological studies have not been conducted, survey data indicate that lifetime prevalence rates for at least one nocturnal panic attack in individuals with PD are as high as 71\% [74, 76-79]. Based on one 
report with a small sample, it appears that nocturnal panic attacks in PD emerge during the transition from lighter to deeper NREM sleep, either in visually scored N2 preceded by EEG slowing (indicating a transition to deeper sleep) or in early N3 sleep [80]. There is some evidence to suggest that individuals with PD and nocturnal panic suffer from higher rates of depression [80] and suicidality [81] compared with individuals with PD without nocturnal panic. Individuals with nocturnal panic may also suffer from more-frequent daytime panic attacks and greater somatic symptoms during daytime attacks [76]. These findings have led researchers to postulate that nocturnal panic attacks may represent a more severe variant of PD.

Clinical features of sleep disturbance: specific phobia and SAD Limited research exists examining sleep disturbance in phobias and SAD [41]. To summarize, two studies using community samples found that the presence of phobias and SAD was associated with increased likelihood of having sleep disturbances, as measured by the PSQI [40] and WHO Composite International Diagnostic Interview (WHO CIDI) [82].

Clinical features of sleep disturbance: OCD

Several studies have examined subjective sleep disturbance in adults with OCD. One study found no differences in sleep parameters when comparing an OCD and controls [83]. The second study found greater subjective reports of sleep disorders and poorer subjective sleep quality in individuals with OCD compared with healthy controls [84]. Similar to other anxietyrelated disorders, greater OCD symptom severity may be associated with greater sleep disturbance severity; a crosssectional study demonstrated that short sleep duration predicted OCD in a Korean community sample (Park et al. [85]) and another cross-sectional study found that greater OCD symptom severity was associated with shorter sleep duration and decreased sleep efficiency in 13 adults with an OCD diagnosis [86]. Conversely, Marcks et al. [87] failed to find an association between OCD diagnosis and sleep disturbance in a sample of primary care patients. In a nationally representative sample, Cox and Olatunji [41] found that individuals reporting insomnia symptoms reported increased OCD symptoms relative to those who did not.

\section{Obstructive sleep apnea}

Recently, there has been a growing interest in the relationship between OSA and anxiety-related disorders, particularly PTSD. OSA is a sleep disorder characterized by repeated upper airway obstruction during sleep, leading to breathing interruptions and sleep fragmentation [88]. The underlying pathophysiology of OSA is multifactorial and complex and may vary considerably across individuals. Important components of OSA pathophysiology include upper airway anatomy, the ability of upper airway dilator muscles to respond to respiratory challenges during sleep, arousal thresholds during sleep, the stability of the respiratory control system, and state-related changes in lung volume [89, 90]. Major risk factors for OSA include obesity, older age, male sex, smoking, and use of central nervous system depressants (e.g., alcohol). The apnea hypopnea index (AHI) is the number of apneas or hypopneas recorded per hour of sleep. The AHI is often used as a diagnostic tool and is a marker of OSA severity.

OSA prevalence rates appear to be higher in PTSD samples compared with the general population [91-99]. Greater PTSD symptom severity increases the risk of screening positive for OSA [91]. On the other hand, including both veteran and non-veteran studies, prevalence rates of OSA in PTSD vary considerably [92, 100-105]. Differences may result from differences in diagnostic criteria and sample characteristics such as sample size, sex, age, ethnicity, and health status compositions. It is also important to note that some studies generated prevalence estimates from samples specifically recruited for OSA-high-risk features (e.g., sleep disturbance), which is likely to artificially inflate prevalence estimates.

There is insufficient evidence to conclude that OSA rates in nonPTSD anxiety disorders differ relative to controls. One crosssectional study using data from outpatient records of the Veteran Health Administration found that an OSA diagnosis increased the odds of having an anxiety disorder diagnosis in veterans [106]. With respect to $\mathrm{PD}$, the majority of work elucidating associations between PD and OSA has been limited to case studies [107-109], studies with small sample sizes $[110,111]$, and studies without an appropriate control group [111]. To our knowledge, only one study has examined OSA prevalence rates in PD and GAD; this study was conducted in a community sample of OSA-high-risk participants and reported an OSA prevalence rate of $58.8 \%$ in PD and $57.1 \%$ in GAD [112]. Another study demonstrated that individuals with PD demonstrated significantly greater rates of microapneas during sleep than controls [110].

In summary, although high rates of OSA are reported in PTSD, these are not convincingly demonstrated in other anxiety-related disorders, and the mechanism explaining the relationship between OSA and PTSD remains to be clarified [98]. The increasingly high rates of obesity in veterans [113] and people with PTSD [114] may be a major factor contributing to this surge in OSA prevalence.

\section{PHYSIOLOGICAL AND FUNCTIONAL NEUROANATOMICAL FEATURES OF SLEEP DISTURBANCE IN ANXIETY-RELATED DISORDERS}

A number of polysomnography (PSG) studies along with a few neuroimaging studies have begun to identify physiological and functional neuroanatomical features that may underly the subjective complaints of sleep disturbance in anxiety-related disorders. We focus here on polysomnography-based studies, and also include a brief presentation of neuroimaging and neuroendocrine research. Of note, we do not discuss actigraphy-based findings in detail here, and instead focus on direct measures of physiology during sleep in anxiety disorders. Overall, actigraphybased findings demonstrate objective sleep abnormalities in anxiety-related disorders, although there are inconsistencies in results within and across disorders $[41,84]$.

Sleep physiological findings: PTSD

Several studies, including two meta-analyses, now provide convincing evidence of PSG-measured sleep abnormalities in PTSD [41, 115, 116]. In a meta-analysis incorporating 20 studies, Kobayashi and colleagues found that PTSD was associated with more stage N1 percent, and lower SWS percent as compared with a mix of controls, including healthy subjects, trauma-exposed, or non-exposed [116]. Authors of a more-recent meta-analysis, which only incorporated 13 studies owing to more-restrictive inclusion criteria, and which incorporated new studies not reviewed by Kobayashi et al., also reported less SWS duration in PTSD as compared with a mix of controls [115]. The latter study found no differences in N1 or N2 sleep duration. On the other hand, they report that PTSD was associated with a reduction in PSG sleep continuity as compared with controls, as defined by a composite measure including sleep latency, sleep efficiency, and/or number of awakenings [115].

Needless to say, the above meta-analyses were performed in the context of inconsistencies in the literature. Some clinical studies observed no differences in PSG-based sleep measures in PTSD vs. control groups (all trauma exposed) [99, 117], whereas other studies have found reduced SWS, increased stage 1 sleep, reduced sleep efficiency, reduced sleep duration, and/or greater number of awakenings in PTSD relative to trauma-exposed [118] or healthy and depressed controls [119]. Inconsistencies have been attributed to moderating third variables such as age, sex 
60

[116, 120], comorbid diagnoses, and substance use disorders [116]. For example, the Kobayashi et al. meta-analysis also found an effect of PTSD on total sleep time (shorter in PTSD) in studies with male samples only. Finally, laboratory-based studies of PTSD subjects suggest that PTSD subjects may in fact sleep better in the supervised environment of the sleep laboratory. This may obfuscate differences in objective sleep quality in PTSD relative to controls.

In terms of REM sleep, both of the above-cited meta-analyses reported that PTSD was associated with greater REM density (REMD), an index of eye movements during REM sleep, than controls $[115,116]$. The implications of REMD are not entirely clear, although some research indicates that REMD is an indicator of phasic hyperarousal during sleep. Interruptions or fragmentation of REMS have also been reported in PTSD and/or trauma survivors relative to controls [56, 121, 122], including PTSD-positive vs. PTSD-negative veterans [56], and PTSD-positive vs. normal healthy civilians [121]. In a subsample of a representative communitybased sample Breslau et al. reported that more frequent arousals from REMS was associated with a history of lifetime PTSD [122]. Mellman and colleagues [123] have also found that REMS fragmentation in the early aftermath of trauma predicted subsequent development of PTSD symptoms. Complicating the REM/PTSD story, Mellman et al. [124] also reported that duration of PTSD was associated with higher REM percent, longer REM duration, and shorter REM latency. Mellman et al. [124] suggested that the linear association between REM parameters and the course of PTSD may reflect an adaptive process in chronic patients, as REM sleep is implicated in emotional processing. On the other hand, Ross suggests that this "reconstituted" REM sleep in chronic PTSD is actually pathological [125]. Richards et al. found longer duration of REMS in PTSD relative to exposed controls in women only [120]. Studies in animal models have fairly consistently demonstrated alterations in REMS in rodent models of acute and chronic stress, which may be relevant across anxietyrelated disorders, but these have included both disruptions and prolongations of REMS. Altogether, these findings provoke ongoing interest in REMS but limited definitive conclusions about patterns of disturbance [126-131].

Although few neuroimaging studies have been performed to understand sleep disturbance in anxiety-related disorders, a number of studies have been conducted in PTSD. For example, Germain and colleagues [132] performed FDG PET imaging in military veterans with and without PTSD and found hypermetabolism in both wake and REM sleep in PTSD-positive, relative to PTSD-negative subjects, in regions involved in emotion expression and emotion regulation. These included the mPFC, the (right) amygdala, and hippocampus as well as brainstem regions overlapping with the raphe nuclei and (right) locus coeruleus. They also saw elevated activity in the thalamus in PTSD relative to controls. In another study, Germain and colleagues reported hyperactivity in NREMS in PTSD relative to control subjects, as well as increased elevation in NREMS relative to wake, in PTSD more than controls [29]. Although these reports indicate hyperarousal of both cortical and subcortical brain regions during sleep, other studies contribute to a more complex story with less straightforward interpretation [30, 133, 134].

Other approaches have been utilized to examine the evidence for physiological hyperarousal during sleep in PTSD by measuring peripheral indicators of neuroendocrine activity. Indices of hyperarousal such as nighttime excretion of norepinephrine metabolites and hypothalamic-pituitary-adrenal (HPA) axis activity have indeed been associated sleep disturbance in PTSD [135-138].

\section{Sleep physiological findings: GAD}

Studies examining physiological sleep characteristics in GAD are limited and therefore it is difficult to draw conclusions [41]. Nonetheless, PSG-based differences in GAD vs. controls are reported. compared with control groups, individuals with GAD exhibit decreased sleep duration [139, 140], increased sleep onset latency [139], increased wake after sleep onset and reduced sleep efficiency [140], reduced NREM N2 sleep and reduced SWS [140]. Two studies found no differences in objective sleep efficiency between GAD and controls [139, 141]. A separate study found that individuals with GAD exhibit increased REM latency compared with individuals without GAD [141].

Sleep physiological findings: PD

Objective reports of PSG sleep abnormalities in PD are less clear. Some studies have found that individuals with PD exhibit decreased sleep efficiency [80, 142-144], increased onset latency $[80,142,143]$, and reduced sleep duration $[78,80]$ compared with controls. Another study found no differences in PSG measures of sleep disturbance, despite subjective differences in sleep quality across a PD and control group [73]. A recent meta-analysis based on four studies concluded that PD is associated with poorer sleep efficiency and marginally significant longer sleep onset latency when compared with individuals without PD [115].

An older and small body of research exists examining sleep physiological characteristics in PD [41]. The existing literature has demonstrated decreased REM latency [142, 145], decreased number of REM periods [80], decreased REM density [145], increased stage $1 \%$ [146], decreased stage 2 duration [143], and deceased SWS \% [78, 144]. Contrarily, Dube et al. [147] found no differences in sleep physiology when comparing PD to healthy controls.

\section{Sleep physiological findings in OCD}

A small number of studies have found differences in NREM sleep parameters in individuals with OCD compared with healthy controls [41]. More specifically, studies have reported decreased sleep duration $[148,149]$, reduced sleep efficiency $[149,150]$, and greater number of nocturnal and early morning awakenings [148$150]$ in individuals with OCD compared with healthy controls. Studies also indicate increased N1 sleep [148], decreased N2 sleep [148], and decreased SWS [148, 151] in individuals with OCD. There is also evidence of altered REM parameters in OCD, though results are mixed. Some studies have demonstrated reduced REM latency [148], higher REM density in the first REM period [149], and a trend towards reduced REM efficiency $(p=0.06$; [148]) in individuals with OCD. In contrast, other studies have found no differences in REM parameters between OCD and control groups $[86,150]$. A recent meta-analysis that combined data from PSG, self-report and observer reports found that sleep duration was also reduced in OCD relative to healthy controls [152].

\section{Summary of Sleep Physiological Findings}

In summary, published sleep-physiological studies overall indicate hyperarousal during sleep in anxiety disorders, with effects on sleep architecture and continuity that may impact some important functions of sleep. It is possible that publication bias has reduced the number of negative published results, favoring publications detecting differences between anxiety disorders and controls. On the flip-side, the generalized but subtle pattern of disruption may indicate that measuring hyperarousal during sleep is more complex than current methods are able to discern, and more sophisticated methods of measurement and analysis such as quantitative EEG analysis [120, 153-155] and brain imaging during sleep [156] are indicated.

EVIDENCE FOR A BIDIRECTIONAL RELATIONSHIP BETWEEN SLEEP DISTURBANCE AND ANXIETY-RELATED DISORDERS Most studies demonstrating high levels of sleep disturbance in anxiety-related disorders are cross-sectional, therefore the causal relationship between sleep disturbance and anxiety-related 
disorders cannot be discerned. However, a number of studies strongly indicate that sleep disturbance enhances risk for future anxiety-related disorders, especially PTSD, and there is also empirical evidence from human studies demonstrating that that anxiety-related disturbances can precede and increase risk for sleep disturbances. We briefly review studies indicating this bidirectional relationship here.

Prospective studies have convincingly demonstrated that sleep disturbance both prior to and following trauma is an important predictor of subsequent PTSD development [117, 157, 158]. Van Liempt and colleagues [159] found that nightmares, although not insomnia symptoms, prior to military deployment predicted PTSD subsequent to deployment. Furthermore, treatment research indicates that treatment of insomnia using standard cognitivebehavioral therapy for insomnia (CBT-I) results in an improvement in daytime PTSD symptoms [160, 161], indicating that insomnia may be a driver of PTSD symptoms, which, when treated, results in a diminution of PTSD symptoms. A recent meta-analysis by Hertenstein et al. [162] also demonstrated that insomnia symptoms prospectively predicted anxiety symptoms in the short-term (12-24 months) and long-term (>24 months). In the other direction, Wright et al., cited above, found that PTSD symptoms 4 months after trauma did not predict insomnia symptoms at 12 months. In contrast, an interesting recent study used ecological momentary assessments of PTSD and insomnia symptoms and demonstrated that daytime anxiety predicted (temporally preceded) poorer sleep quality and efficiency, even when controlling for baseline PTSD symptoms [163]. This study also found that daytime PTSD symptoms and fear of sleep predicted subsequent nightmares. Findings from a large community-based sample of adolescents also showed that having any anxiety disorder was prospectively associated with an increased risk of insomnia [164]. In that study, insomnia did not predict the future development of anxiety disorder. Although our review is mostly focused on sleep and anxiety in adults, research in children and adolescents, when these disorders first present, may be particularly informative with respect to the chicken-andegg question.

To our knowledge, studies that prospectively examine the relationship between sleep and non-PTSD anxiety disorders is limited. As described above, in a study in adolescents aged 13-17, anxiety disorder predicted future insomnia, whereas insomnia did not predict future anxiety [164]. One prospective study utilizing self-report measures found that sleep disturbance was associated with anxiety symptoms (though not GAD specifically) or a diagnosis of PD 4 years later [165]. CBT for GAD reduces sleep disturbance symptoms, but one study demonstrated that $33 \%$ of individuals had an insomnia disorder diagnosis and $63 \%$ had clinically significant sleep disturbance following treatment, indicating that insomnia is not a result of GAD alone, and/or that insomnia that may have resulted from GAD has taken on a life of its own, and is therefore unresponsive to GAD treatment. Several laboratory studies using total sleep deprivation paradigms have demonstrated that sleep deprivation increases the vulnerability to subsequent daytime panic attacks $[166,167]$. It is not clear how these experimental results generalize to sleep disruption in the real world. Additional work has demonstrated that sleep disturbance symptoms persist following successful PD treatment $[168,169]$, which suggests that sleep disturbance is not a secondary symptom of PD or that sleep disturbance has become independent of original PD precipitants.

\section{EXPLAINING THE RELATIONSHIP BETWEEN SLEEP DISTURBANCE AND ANXIETY-RELATED DISORDERS: PSYCHOLOGICAL AND PSYCHOPHYSIOLOGICAL MODELS}

Although the extant research indicates that sleep disturbances and anxiety-related processes mutually influence each other, the mechanisms through which this occurs is not yet clear. The dominant psychological and psychophysiological models of insomnia disorder certainly provide clues about these mechanisms.

Insomnia disorder is a sleep disorder characterized by subjective difficulties with sleep onset and/or sleep maintenance, despite adequate opportunity to sleep, with associated daytime impairment or distress [46]. A wealth of research has advanced our understanding of insomnia as a condition resulting from and reinforced by maladaptive, sleep-focused cognitions and maladaptive behaviors and coping strategies. Genetic and biological/ physiological traits may predispose individuals to the development of insomnia, and physiological disturbances also result from maladaptive cognitions and behaviors. The interaction of cognitive, behavioral, and physiological factors all contribute to a vicious cycle of sleep disturbance and associated distress. The profound value of these models and testimony to their validity is that the CBT-I that is based on these models is the first-line therapy for insomnia disorder, with proven efficacy in insomnia alone and comorbid with a multitude of psychiatric and medical conditions [170, 171]. CBT-I has also demonstrated benefits for anxiety symptoms in several studies [161, 172].

The diathesis-stress model states that there are three interrelated and sequential factors involved in the pathogenesis of insomnia: predisposing, precipitating, and perpetuating factors [173]. Predisposing factors are individual genetic, physiological, or psychological traits that produce differing levels of susceptibility for developing insomnia (e.g., sex, chronotype, genetic polymorphisms affecting arousal regulation or cognition). Precipitating factors are physiological, environmental, or psychological stressors that trigger the onset of disrupted sleep patterns and include factors ranging from physical injury, work-related stress, or a traumatic stressor. They may or may not be psychological in nature. Perpetuating factors are maladaptive strategies for coping with disrupted sleep that contribute to insomnia symptoms and result in chronic insomnia if sustained. They generally reflect efforts to compensate for insufficient nighttime sleep and are comprised of behaviors such as sleeping in on weekends, taking daytime naps, and increasing time in bed in an effort to obtain the desired amount of sleep. The perpetuating influence of the latter behaviors is explained by the stimulus-control model proposed by Bootzin [174], and is anchored in classical conditioning theory. Increased time in bed in the absence of actual sleep eventually results in increased frustration in bed (i.e., cognitive and physiological arousal), which effectively reduces the likelihood of sleep and results in a further perpetuation of wakefulness rather than sleep. Their perpetuating effect is enhanced by the fact that the compensatory mechanisms result in efforts to sleep at physiologically suboptimal times (i.e., in the absence of homeostatic and circadian pressures to sleep).

Cognitive models of insomnia focus on thoughts, beliefs, and/or feelings that may interfere with sleep and lead to maladaptive coping behaviors [175]. According to cognitive models of insomnia, excessive worry and rumination lead to arousal and distress, all of which precipitate and perpetuate insomnia symptoms [175]. Harvey hypothesized that insomnia is driven by inappropriate worry about sleep and sleep-related consequences. This worry subsequently leads to physiological arousal, selective attention to sleep-related threats (e.g., ambient noise), and the adoption of maladaptive safety behaviors. Another cognitive model proposed by Espie proposes that psychological and/or physiological stress leads to selective attention towards stressors, which inhibits the natural "de-arousal" that is necessary to initiate sleep [176].

Riemann and colleagues have advanced a hyperarousal model of insomnia that incorporates the elements of the above models while also emphasizing a fundamental contribution of genetic and physiological vulnerability to sleep/wake regulatory problems and 
62

to hyperarousal at a physiological as well as cognitive level. In their words, they conceptualize insomnia as "a final common pathway resulting from the interplay between a genetic vulnerability for an imbalance between arousing and sleep-inducing brain activity, psychosocial/medical stressors and perpetuating mechanisms including dysfunctional sleep-related behavior, learned sleep preventing associations, and other cognitive factors like tendency to worry/ruminate" [177]. They cite evidence indicating that hyperarousal processes from the molecular to higher system levels play a role in the pathophysiology of insomnia [177]. Several other researcher groups have demonstrated that hyperarousal in insomnia exists in cognitive (e.g., sleep-related worries [178, 179], somatic/ physiologic (e.g., increased heart rate; $[178,179])$, and cortical (e.g., high frequency EEG) [179] domains. Riemann et al. [177] have pointed out that while standard visual scoring of EEG has often failed to show differences between insomnia and controls, more sophisticated approaches to EEG analysis are pointing to subtle and dynamic features that distinguish good from poor sleepers. These methods are now often used in insomnia disorder research but have only been used in a small number of studies of sleep in anxietyrelated disorders $[120,153,154]$.

Altogether, research on insomnia provides strong evidence for an interplay between biological predispositions, sleep-disrupting life events, and maladaptive coping behaviors that result in cognitive and physiological hyperarousal, that then perpetuate the cycle of sleep disturbance. The model described by Riemann et al. proposes both cognitive-behavioral and neurobiological pathways through which biological and cognitive risk factors may lead to insomnia as well as anxiety disorders, that then positively reinforce sleep-disturbance in a vicious-cyclic pattern.

Applicability of the dominant insomnia models to PTSD

Consistent with the above models, a traumatic event amounts to a classic and severe insomnia "precipitating factor" that may result in disrupted sleep owing to a variety of factors [180]. From a psychological perspective, nighttime arousal that occurs immediately following trauma may be adaptive in settings of increased predatory threat (e.g., combat), or owing to one's increased vulnerability when asleep [181, 182]. Unfortunately, whether initially adaptive or not, compensatory behaviors (e.g., naps) to compensate for nighttime sleep deficits will result in mistimed sleep, and therefore wakefulness in bed even in the absence of psychological distress, and thereby contribute to conditioned arousal. On top of this, nighttime trauma-related psychological distress, such as distressing trauma memories and ruminations, and frightful awakenings occurring either spontaneously or from nightmares, are likely to contribute to conditioned arousal in the sleep environment. Although the insomnia models emphasize sleep-related cognitions as a precipitant of hyperarousal, the essential ingredient is that worry in the bedroom, at night, and in the sleep environment precipitates hyperarousal. In this context, the application of insomnia models to PTSD is therefore straightforward, and the success of standard CBT-I for treating sleep disturbance in PTSD provides strong support for its relevance to PTSD [160].

Fear of sleep is common in PTSD and distinguishes sleep-related cognitions in PTSD from insomnia (in which individuals desire rather than fear sleep). Evidence suggests that fear of sleep is driven by both fear of having a nightmare and fear of the vulnerability that occurs during sleep [163, 183-185]. Fear of sleep may result in individuals avoiding sleep and/or in difficulties with sleep initiation. Some studies have elucidated an association between fear of sleep and insomnia symptoms [163, 185, 186], whereas other studies have found no such association [183]. One highly credible explanation for the latter findings is that over time, insomnia is no longer driven by fear of sleep and is instead maintained by a pattern of learned maladaptive coping behaviors that are no longer dependent on the initial precipitating causes [183].
Applicability of the dominant insomnia models to GAD Cognitive and hyperarousal models of insomnia may be particularly applicable for insomnia pathogenesis in GAD. A cardinal feature of GAD is excessive cognitive activity, specifically worry and rumination. Although not performed specifically in GAD subject, one study demonstrated that sleep-related rumination was associated with both trait and state arousal in insomnia disorder [187]. Several studies have also demonstrated associations between worry, rumination, and sleep disturbance, though most of these studies are limited to undergraduate samples and results may not be generalizable [188-192]. On the other hand, some studies examining trait worry and rumination failed to find significant associations with sleep disturbance [193]. O'Kearney et al. [194] found that worry was associated with insomnia symptoms as measured by the ISI, but not specific sleep continuity parameters.

Applicability of Dominant Insomnia Models to PD

Within the insomnia model framework, nocturnal panic attacks can be considered a precipitating factor for insomnia, disrupting sleep, and contributing to sleep-environment arousal and fear of having another panic attack [79]. Repeated nocturnal panic attacks produce conditioned fear (e.g., hyperarousal) and avoidance of sleep [79], which results in the adoption of maladaptive sleep-related behaviors (e.g., sleeping in brightly lit and very loud environments to obtain rest but avoid deep sleep). Conditioned fear, avoidance of sleep, and maladaptive safety behaviors are perpetuating factors and maintain insomnia symptoms in the absence of nocturnal panic attacks.

Two studies have demonstrated the presence of insomnia symptoms in the absence of nocturnal panic attacks, suggesting that other factors may be involved in insomnia pathogenesis in PD $[72,195,196]$. Anxiety sensitivity-a tendency to experience excessive fear of anxiety-related sensations based on the fear that these sensations are harmful [197] has been associated with sleep onset difficulties in PD [198]. Anxiety sensitivity may amplify the conditioned hyperarousal characteristic of insomnia by leading to a hyper-focus on physiological symptoms. Related to this, two studies manipulating pre-sleep appraisal of sleep physiology support this theory by demonstrating that individuals with PD and nocturnal panic report more distress when they believe that physiological signals are abnormal $[199,200]$.

Contrasting with this idea of anxiety sensitivity, Craske and Tsao [74] also propose that nocturnal panic in PD is driven in part by fear of loss of vigilance. Evidence cited to support this model included studies demonstrating higher physiological and selfreported emotional reactivity to relaxation and imagery exercises, greater self-reports of panic attacks following relaxation and fatigue, and greater self-reported discomfort during relaxation exercises in individuals with PD and nocturnal panic compared with individuals with PD without nocturnal panic [80, 201, 202].

Applicability of dominant insomnia models to OCD

A recent study exploring associations between insomnia symptoms and OCD symptoms in an undergraduate sample found that insomnia symptoms were associated with obsessions, but not compulsions [203]. This finding suggests that similar to other anxiety-related disorders, hyperarousal and cognitive activity may be driving insomnia in individuals with OCD as obsessions are characterized by heightened arousal and compulsions serve to alleviate anxiety.

\section{Summary}

In summary, the above-described findings implicate fear, threat, and anxiety-related cognitions occurring at night or in the sleep environment, or in a trait-like fashion and therefore likely to occur at night, in sleep disturbance associated with anxiety-related disorders. Whether they are focused on anxiety about missed 
sleep (insomnia) or any other range of concerns may be less important than the fact that they reflect overall cognitive hyperarousal in the sleep environment and as such result in physiological arousal as well as all the downstream compensatory and conditioning phenomena typical of insomnia disorder. Overall, this reflects a transdiagnostic process, consistent with a transdiagnostic model of insomnia relevant across psychiatric disorders.

\section{AFFECTIVE AND COGNITIVE NEUROSCIENCE OF SLEEP INFORM THE UNDERSTANDING OF SLEEP DISTURBANCE IN ANXIETY- RELATED DISORDERS: EMOTION REGULATION AND EMOTIONAL MEMORY}

Insomnia models satisfactorily explain many of the subjective sleep complaints and insomnia manifestations per se in anxietyrelated disorders, but they do not fully or explicitly incorporate the effects of objective disruptions of sleep integrity on two aspects of brain functioning that may be elemental to the disorders discussed here: emotion regulation and emotional memory.

\section{Sleep disturbance and emotion regulation}

Emotion regulation generally refers to the ability to modulate emotional experiences in a way that is responsive to the context of a situation as well as an organism's long-term objectives [187]. In anxiety disorders, emotion-regulatory problems manifest as the maladaptive expression of fearful and anxious emotions and emotional responses. Emotion regulation may be considered an aspect of cognitive executive function [4]. The prefrontal cortex (PFC) has been demonstrated to play an important role in emotion regulation [204, 205], and the frontal lobes may be particularly vulnerable to sleep loss. Gruber and Cassoff [4] recently published an informative review of empirical findings and a conceptual framework for understanding the importance of sleep for executive functioning and emotion regulation more specifically.

Related to this, it has been reported that deficits in cognitive inhibition (e.g.,"tuning out" irrelevant stimuli) [206] and emotion regulation [187] were associated with sleep-related rumination in individuals with insomnia disorder. Particular to GAD, one study found that difficulties with emotion regulation mediated the association between GAD diagnosis and insomnia symptoms [207]. A recent study in PD found that poorer performance on a cognitive inhibition task was associated with longer sleep onset latency and reduced sleep quality in individuals with PD [208].

A recent imaging study provides neurobiological evidence for the role of emotion regulation in the anxiety-sleep relationship. Pace-Schott et al. found that compared with GAD and insomnia disorder groups, a good sleeper group exhibited greater restingstate functional connectivity between the left amygdala and a bilateral region of the rACC. As described earlier, the $\mathrm{rACC}$ is part of a prefrontal network believed to exert top-down control over amygdala activity and may constitute an emotion-regulatory circuit. These findings support the idea that deficits in emotion regulation may play a role in both anxiety and insomnia pathogenesis [209].

These studies do not explain causal relationships, and do not necessarily distinguish between objective sleep disruption and insomnia symptoms/disorder per se, yet they provide evidence that deficits in cognitive functions, which may result from sleep disturbance or be a manifestation of an anxiety disorder, are mechanistically involved in the sleep-disturbance-anxiety disorder cycle.

Sleep and memory: relevance to anxiety-related disorders A sizable body of research now provides strong evidence that sleep has an important role in memory consolidation [10, 11, 210213]. The "off-line" state of sleep provides a unique opportunity to consolidate information considered relevant to the organism through the reinforcement of key synaptic connections in the relative absence of environmental inputs and high energyexpenditure needs [10]. Although the most-compelling evidence currently points to NREMS as the major driver of sleep-dependent memory consolidation, both NREMS and REMS have been shown to be relevant for consolidation of memory of various sorts $[11,210,214]$. Research on sleep in anxiety-related disorders, and PTSD in particular, has honed in on REMS as an important player in the processing of fearful, threatening, or distressing experiences in a way that results in selective and adaptive consolidation of emotional memories [215, 216].

Fear conditioning and related protocols in both animal models and human subjects constitute the dominant approach for studying the role of sleep and REMS in emotional learning relevant to anxiety-related disorders [13, 217, 218]. Fear conditioning involves the transformation of a neutral stimulus, such as a neutral visual image or context, into a frightening stimulus through its repetitive pairing with an aversive experience, such as an electric shock. Via classical conditioning, the neutral stimulus becomes a conditioned stimulus eliciting a fearful emotional response, usually measured using electrophysiology techniques such as skin conductance response or electromyography responses in humans. Once established, conditioned fear can be extinguished through subsequent repetitive presentation of the conditioned stimulus in the absence of the aversive stimulus. Modifications of the simplest fear-conditioning protocols exist, including addition of "safety" stimuli that are never paired with an aversive stimulus, the responses to which can be compared with conditioned fear responses to assess the subject's ability to discriminate between fear and safety cues. Neuroimaging research indicates that fear extinction is mediated in large part by the mPFC, in particular the ventromedial PFC and rACC, which are structures implicated in anxiety-related disorders $[13,219]$.

Although findings are not entirely consistent across studies, which utilize different measures of REMS consolidation and/or different outcome measures, recent findings in humans indicate that intact REMS is important for adaptive extinction of conditioned fear, including retention of extinction over time, and the ability to distinguish between safety and fear signals $[218,220]$. This has important implications for psychotherapies since extinction and safety learning are psychological models underlying psychotherapy for anxiety. Although not focused on REMS, there is indeed some evidence indicating that sleep may enhance the efficacy of phobia and SAD treatment [221]. Exposure therapy followed by a period of sleep is associated with decreases in heart rate [222], skin conductance [222], and self-reported fear [223] when confronted with the feared stimulus compared with sessions followed by wake. Poorer sleep at baseline was associated with poorer outcome following CBT treatment for SAD and more restful sleep following sessions was associated with a greater reduction in SAD symptoms [224].

Although fear conditioning is a form of implicit emotional learning, some researchers have also found REMS associations with declarative forms of emotional memory $[14,225,226]$. These studies may be most pertinent to PTSD, the symptoms of which revolve around a traumatic emotional memory and include persistent, intrusive emotional memories [227]. For example, Walker et al. found that higher REMS duration correlated with enhanced emotional, vs. neutral, recall in a nap paradigm involving recall for emotionally distressing vs. neutral images [225]. Walker and others have proposed an emotional processing function to REMS, wherein REMS enhances the declarative recall of emotional memories while diffusing the negative emotional valence [216]. On the other hand, several studies reported findings indicating that REMS in healthy subjected either heightened, or did not affect, the emotional tone associated with memories [15, 226, 228, 229]. Furthermore, a REM-deprivation study found that selective REMS deprivation did not impact emotional memory. Another study found that while sleep after 


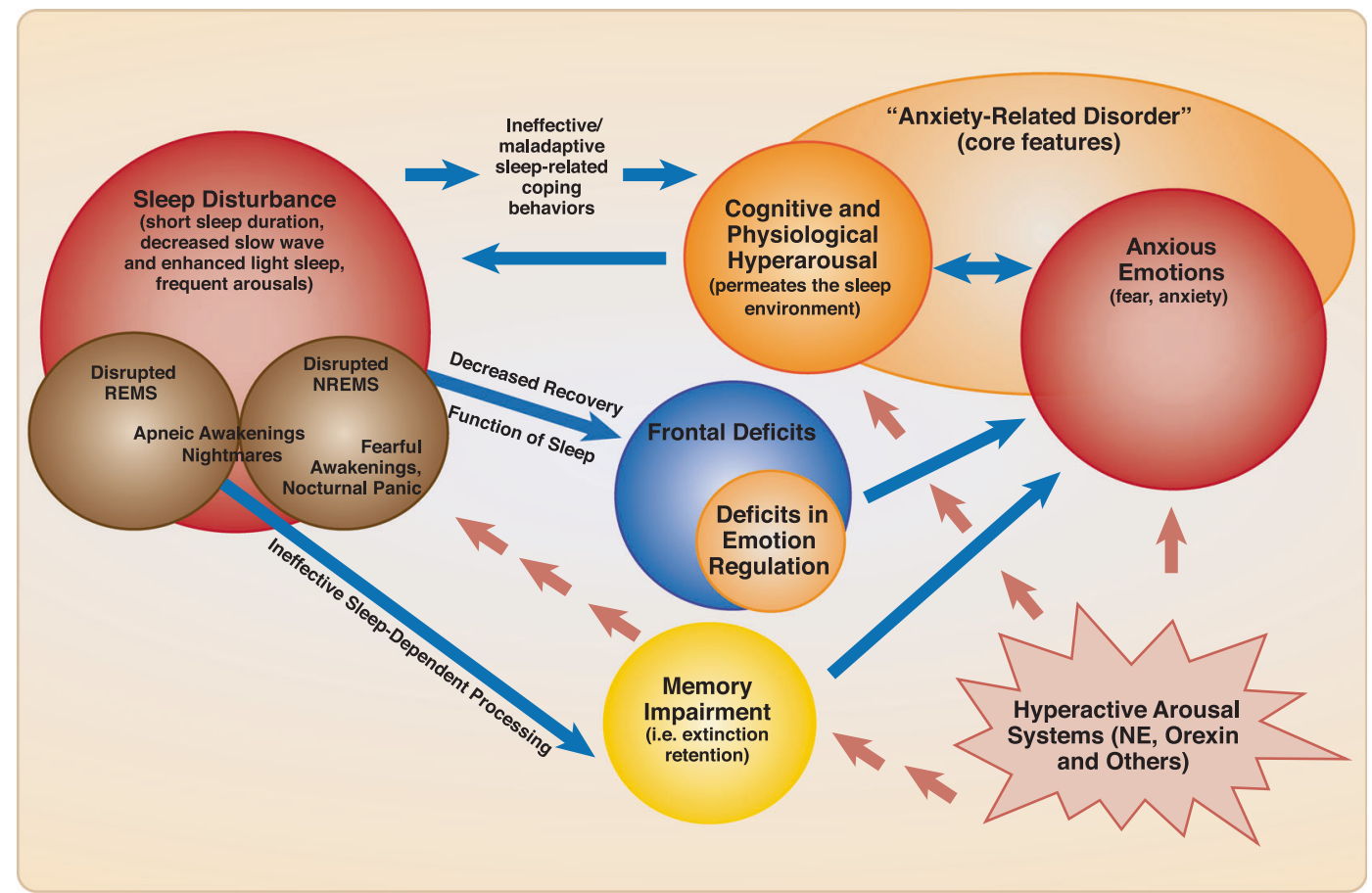

Fig. 4 Schematic diagram including the psychological and neurocognitive factors that link sleep disturbance and anxiety-related disorders in a bidirectional fashion. Sleep disturbance, regardless of cause, may result in maladaptive sleep-related compensatory behaviors, resulting in sleep timing that is out-of-synchrony with sleep drive, and therefore wake time in bed. This results in hyperarousal (i.e., cognitive and emotional) in the sleep environment, promoting a vicious cycle of sleep disturbance, compensatory behaviors, hyperarousal cognitions and anxious emotions. Sleep disturbance also has deleterious effects on cognitive functions, due to insufficient sleep-dependent recovery of neural substrates that carry out said functions during wake; this results in widespread deficits including deficits in emotion regulation (i.e., reduced ability to modulate anxious emotions). Sleep disturbance also disrupts processes thought to be carried out during sleep, such as adaptive memory functions (e.g., fear extinction retention; safety learning); this also promotes or maintains anxiety/fear emotions. Anxious emotions and cognitive hyperarousal are mutually reinforcing, and feed back into the sleep disturbance cycle. The specific role of REMS and NREMS in recovery and sleep-dependent processes is a topic of investigation. The nature of mechanistic connections between anxiety-related events such as nightmares and panicked awakenings, and NREM or REM sleep-dependent processes, remains to be demonstrated with empirical evidence. At the very least, their promotion of cognitive hyperarousal and anxious emotions can be inferred from their contribution to sleep disruption and the fearful emotions they generate. A neuromodulatory milieu involving heightened arousal signals, e.g., norepinephrine, and/or hypocretin, drives and underlies this relationship

learning did enhance emotional memory recall relative to a nonsleep group, selective REM deprivation had no impact on negative vs. neutral recall [230]. These varied findings leave researchers with many unanswered puzzles, and suggest that REM/NREM distinctions may be inadequate for pinpointing the complexities of sleep effects on emotional memory.

Sleep spindles deserve brief mention because sleep spindles reflect memory consolidation processes involving the coordinated oscillations of the thalamo-cortical circuitry, the hippocampus and the cerebral cortex that together result in the transfer of information from temporary storage in the hippocampus to more durable cortical storage [211, 231]. In a recent laboratory study in which subjects viewed a trauma film prior to either overnight sleep, a day of wake, or sleep deprivation, Kleim and colleagues [232] found that greater N2 sleep and more parietal spindles $(13-15 \mathrm{~Hz})$ in the sleep group were associated with fewer intrusive memories in the week following the laboratory exposure. They also found that increased N1, wake-after-sleep-onset, and REMD were associated with higher intrusions, and that sleep was protective relative to both the other groups. Although few studies have examined spindles in emotional memory, these findings may be relevant in the context of anxietyrelated disorders, especially PTSD.

\section{WHAT ABOUT NIGHTMARES?}

Nightmares continue to be an elusive and intriguing phenomenon, which have generated a host of theories regarding etiology and possible function. A long history of dream theory based primarily in psychoanalytic work, but also in peer-reviewed dream research, has espoused a psychological, sleep-protective, and/or emotional processing function for dreams [57, 233]. These theories are generally anchored in the notion that emotional dreams are REMS phenomena [234], that REMS is important for emotional processing (Walker, [212]; Walker \& van der Helm, [216]), and that trauma nightmares result from a disruption in the normal emotional processing and consolidation functions of REMS [235237]. These conceptualizations are grounded in compelling reasoning, but require bolstering by empirical evidence. Further, early research increasingly indicates that trauma nightmares are not an exclusively REMS phenomenon; nightmare awakenings occur out of both REM and NREM sleep [59, 238, 239].

In the context of REM sleep models, it has also been proposed that abnormally elevated adrenergic tone during REMS contributes to the distress of REMS dreams and/or awakenings from REMS [240]. Our current understanding of central nervous system pathways involved in sleep and arousal are generally consistent with this hypothesis: norepinephrine-generating locus coeruleus activity is low during normal sleep, and essentially absent during normal REMS. Excess activation of the locus coeruleus could perturb REMS. The hypothesis that excessive adrenergic tone during sleep contributes to nightmares seems reasonable and could apply regardless of sleep stage context. We are not aware of studies that have examined this hypothesis. Results from one recent study demonstrated that reduced respiratory sinus 
arrhythmia during sleep, a measure of parasympathetic tone, was a predictor of nightmare endorsement the following morning [241]. This may indicate that a disruption in the normal balance of parasympathetic vs. sympathetic tone contributes to nightmares, but is not direct evidence of enhanced sympathetic activity.

Despite our limited understanding of the neurobiology underlying the generation of nightmares, nightmares likely contribute to the maintenance of PTSD and sleep disturbance in a manner consistent with the insomnia processes discussed previously. Nightmares are likely to contribute to fear of sleep and sleep avoidance, factors that contribute to altered sleep-related behaviors and insomnia. Short et al. also demonstrated that daytime PTSD symptoms and fear of sleep were prospectively related to nightmares [163]. The effects of repeated activation of trauma-related thoughts during sleep are unknown, but one might speculate that they contribute to reinforcement of memorycontaining neural networks (the PTSD "fear network"), thereby consolidating those memories in association with distressing emotions.

\section{ABNORMAL MOVEMENTS DURING SLEEP: FROM MUSCLE TWITCHES TO NIGHTMARE ENACTMENT}

Abnormal movements during sleep in anxiety-related disorders are also poorly understood phenomena, and are primarily reported in PSTD, as opposed to other anxiety-related disorders. They are grouped together here because they involve abnormal motor behavior during sleep, rather than due to a clearly understood neurobiological overlap. Muscle twitching in the context of REMS atonia is normal phenomena, and there is even evidence that it plays a role in sensorimotor development [214]. However, indications that muscle movements are increased in PTSD relative to normal are consistent with the overall concept of hyperarousal during sleep. Carwile and colleagues proposed that RBD-like phenomena in PTSD (nightmare enactment) may be due to abnormalities in LC NE firing. However, in contrast with the idea that NE activity is elevated in REMS, they propose that it is a high "turnover" and resulting depletion of NE in the LC of PTSD patients that disrupts REM atonia, just like degeneration of LC neurons occurs in neurogenerative diseases [242]. Further research is clearly indicated to better understand the neurobiology of these phenomena and their implications for outcomes in anxiety-related disorders. Regardless of etiology, like other sleep-disrupting events described in this report, they are likely to contribute to the many downstream effects of objective sleep disturbance and the cycle of behavioral, cognitive, and physiological problems that characterize the sleep-anxiety relationship.

\section{SLEEP DISTURBANCE AND ANXIETY-RELATED DISORDERS: UNDERLYING NEUROMODULATORY CONTEXT}

Figure 4 provides a schematic diagram modeling the psychological and neurocognitive factors that link sleep disturbance and anxiety-related disorders in a bidirectional fashion. This relationship occurs in the setting of heightened activity in known neuromodulatory pathways. Animal and pharmacology studies point to important neuromodulators, such as norepinephrine and hypocretin, that play an important role in this dynamic.

Major neuromodulators in sleep-wake regulation and anxietyrelated disorders

Norepinephrine. Norepinephrine is released both peripherally (adrenal medulla) and in the central nervous system as a neurotransmitter and plays a central role in both sleep regulation and anxiety (See Fig. 1). Although its nearly exclusive CNS source, the locus coeruleus, seems to be too small to be captured by classic neuroimaging techniques (although see PET studies above and fMRI findings by Naegeli et al. [243] in PTSD), it is clearly central to anxiety- and fear-related processes in the CNS through its direct innervation of the amygdala and its inhibitory inputs to the VLPO, among a multitude of other projections (e.g., cerebral cortex). Normally, NE activity is high during wake, low during NREMS, and nearly completely quiescent during REMS [244]. Human studies have provided some evidence of elevated sympathetic tone and/or LC activity in anxiety-disordered sleep $[29,136,245]$.

Pharmacological research has studied various noradrenergic blockers for treatment of anxiety-related disorders. For example, the selective alpha-1-adrenergic blocker prazosin has demonstrated benefits in multiple small to medium-sized RCTs for PTSD symptoms and trauma nightmares [246-248]. Disappointingly, a recent large RCT of prazosin for PTSD in veterans demonstrated no benefit relative to placebo and no benefit overall for either PTSD symptoms overall or for nightmares [249, 250]. One recent study indicates that higher baseline systolic blood pressure predicts greater therapeutic response to prazosin for nightmares and sleep disturbance [251]. Doxazosin, a medication with the same alpha-1 blocking effects of prazosin, but with a longer half-life, has shown some benefit in preliminary studies and is currently being studied as an alternative to prazosin [252]. Interestingly, it is thought that doxazosin is less able to cross the blood-brain barrier than prazosin. This raises questions about whether PTSD is associated with greater blood-brain barrier permeability, thus allowing entry of less lipophilic agents, or whether some of the CNS benefits are mediated via peripheral adrenergic nervous system responses. Finally, the alpha-2-agonist clonidine has shown some benefit in PTSD nightmares, however it has not been extensively studied [253].

Serotonin. The neurotransmitter serotonin (5-hydroxytryptophan, $5 \mathrm{HT}$ ) is a neurotransmitter that has an important role in arousal (see Fig. 1) and which also plays an important role in the regulation of anxiety and mood states. It is produced in the dorsal raphe nucleus, which has broad axonal projections including projections to the VLPO (inhibitory) and the cerebral cortex. The arousal effect of $5 \mathrm{HT}$ is mediated in part by $5 \mathrm{HT}-2 \mathrm{~A}$ receptor activity, based on animal studies as well as clinical research demonstrating that agents with high $5 \mathrm{HT}-2 \mathrm{~A}$ blocking effects are sedating (i.e., trazodone, quetiapine, amitriptyline). Agents that selectively inhibit the reuptake of serotonin into neurons (selective serotonin reuptake inhibitors, (SSRI)) and thereby increase $5 \mathrm{HT}$ in the synapse, are first-line agents for anxiety and mood-related disorders, including all of the disorders described in this review. Serotonin levels are high during wake, low during NREMS and at their lowest during REMS [244]. One can therefore see how agents that enhance 5HT across 24 hours (SSRI's and SNRI's, which also block norepinephrine reuptake) might have untoward effects on sleep even if beneficial for anxiety. On the other hand, downregulation of $5 \mathrm{HT}-2 \mathrm{~A}$ receptors with prolonged SSRI administration may be associated with resolution of initial activating effects of these drugs.

Histamine. The role of histamine in sleep/wake circuitry is demonstrated in basic research and is supported by the broad use, though limited controlled research, of various drugs with antihistaminergic properties (e.g., trazodone, quetiapine, amitriptyline, doxepin, diphenhydramine, and cetirizine). It has been proposed that histamine release is under circadian control, and is released earlier than other neurotransmitters. Although circadian disruptions are a major topic that is hardly covered in this review, the possibility the role of circadian timing of histamine release may be relevant to early morning awakening in anxiety-related disorders. Anti-histamines are commonly used off-label for the treatment of anxiety, although evidence for specificity as an anxiolytic, as opposed to a sedating agent, is weak. 
Acetylcholine. Acetylcholine is a neurotransmitter synthesized in several brain regions/nuclei, including the pedunculopontine tegmentum (pons), the lateraldorsal tegmentum (pons) and the basal forebrain and is also an important arousal neurotransmitter. In contrast to the other major neuromodulators described above, CNS cholinergic activity is normally high in both wake and REMS, although decreased in NREMS [244]. Walker and colleagues $[212,216]$ have postulated that elevated cholinergic activity in REMS, combined with maximal suppression of noradrenergic activity during this stage, contributes to the emotion and emotional memory processing function of REMS. Although this remains highly theoretical, Walker and colleagues propose that the memory-promoting function of acetylcholine in REMS acts to consolidate the declarative memories (an adaptive process) in the absence of the NE-promoted emotional "tag".

Several other medications with effects on these neurotransmitters systems have been used to treat sleep disturbance in anxietyrelated disorders. Trazodone, for example, is used widely in public health settings [254] despite limited controlled data on efficacy and is known to block receptors for histamine, serotonin (5HT-2A/ 2C), and alpha-adrenergic receptors, [255]. A recent study of cyclobenzaprine, a tricyclic molecule structurally similar to amitriptyline, which like trazodone blocks histamine, alphaadrenergic, and serotonergic 5-HT2A receptors, was halted during a Phase III development trial because of inadequate separation from placebo for the primary sleep endpoint in military-related PTSD (Clinicaltrials.gov: NCT03062540).

Dopamine. Dopamine, produced in the ventral tegmental area and the ventrolateral periaqueductal gray, is also an important arousal-promoting neurotransmitter. While mostly known for its central involvement in reward neurocircuitry and disorders of addiction, a recent study of quetiapine for PTSD lends some support for dopamine-receptor blockade in the treatment of anxiety-related disorders, and quetiapine is often used off-label for the treatment of insomnia [256, 257]. Quetiapine's broad inhibition of multiple arousal-promoting neurotransmitters, including serotonin (5HT-2A), histamine, and NE (alpha-1) do raise questions about dopamine-specific mechanisms.

Hypocretin/orexin. The hypocretins, also known as orexins, including hypocretin-1 and 2, are neuropeptides with a wellestablished role in promoting and stabilizing arousal [258-260]. Recent publications present the growing evidence implicating the hypocretins in anxiety-related disorders, and the pathways through which they act $[261,262]$. Hypocretin is produced in the hypothalamus, and hypocretin-producing neurons have widespread excitatory projections, including to the main wakepromoting centers (see Fig. 1a), the cerebral cortex, and the amygdala. Overall, the research indicates that hyperactivity of the orexin system contributes to anxiety-related behavior. While most research has been performed in animal models, one recent study demonstrated elevated CSF orexin in patients with PD. An interesting recent study found that hypocretin was related to panicked awakenings in a rodent model. These findings may be highly relevant to understanding nocturnal panic attacks and frightful awakenings in the context of PTSD and PD and deserve further study.

The dual-orexin antagonist suvorexant is FDA-approved for the treatment of insomnia. At present there are no published trials of hypocretin/orexin antagonists in anxiety-related disorders, though a few studies are underway. Theoretically, hypocretin/orexin antagonists would target more-discrete pathways than commonly used GABAergic hypnotics, and ideally would be suitable for individuals who demonstrate abnormally increased hypocretin activity [263]. There is preclinical evidence that orexin antagonism can block stress-induced sleep disturbance but have no effect on baseline autonomic and autonomic activity [264]. The implication is that it is possible to develop compounds that only affect stressrelated over-activation of arousal pathways without interfering with normal activity in healthy individuals.

GABA. GABA receptors are ubiquitous in the CNS and GABAmediated CNS inhibition has a huge role in sleep promotion through specific pathways. On the other hand, endogenous GABA-mediated promotion of sleep involves a targeted suppression of arousal-promoting nuclei, rather than a more global inhibition of the cerebral cortex (See Fig. 1). Despite some theoretical value of targeting GABAergic function in anxietyrelated disorders, the potential for adverse effects (tolerance, dependence, cognitive side-effects, ataxia, respiratory depression) are substantial given widespread distribution of GABAergic inhibitory neurons in the brainstem, cerebellum, and cortex [265]. At least in PTSD, there is also limited evidence of diminished GABA brain activity [266]. Furthermore, GABA also plays a role in arousal through inhibition of inhibitory interneurons in the cerebral cortex. This may contribute to paradoxical disinhibition in response to GABAergic drugs in some individuals. Despite concerns, benzodiazepines have demonstrated benefits for anxiety symptoms, and their use continues to be widespread [267]. There are only a few published controlled studies focused on GABAergic hypnotics in PTSD [268, 269], and the extant evidence is insufficient for recommending a GABAergic approach.

The so-called non-benzodiazepine receptor agonists, or "zdrugs," were developed to specifically target subtypes of the GABA-A receptor, with the aim of reducing adverse effects. One study of eszopiclone, for example, which targets the alpha-3 subunit specifically, provides preliminary evidence of benefit for both sleep disturbance and PTSD [269]. The advantages of z-drugs over the benzodiazepines in sleep disturbance in anxiety-related disorders has not been well-established.

The HPA axis and CRF. Research in humans and rodent models indicate that stress-induced alterations in corticotropin-releasing factor (CRF) and the HPA axis may result in objective sleep quality disturbances, including decreased delta sleep in human subjects, a quantitative measure of slow-wave activity $[137,153]$ and REMS disturbances in rodents [127]. CRF functions as a neurotransmitter in the amygdala, locus coeruleus, and bed nucleus of the stria terminalis, and has an arousing effect on the cortex. Theoretically, CRF antagonism would be a good target for treating anxietyrelated sleep disturbance, however trials of such agents in psychiatric disorders, including PTSD, have been disappointing [270]. Nonetheless, these studies represent an attempt to specifically engage in a target pathway implicated in fear and anxiety states. Future trials with agents that are more specifically targeted to abnormal pathways in PTSD and other anxiety disorders await much-needed advances for understanding the underpinning neurobiology [256].

Other diverse and emerging players: cytokines, adenosine, cannabinoids

Research is beginning to cover the role of multiple other players in sleep-regulatory processes that may be relevant to the relationship reviewed here. Adenosine is a major player in the homeostatic sleep drive (the drive to sleep that increases as a function of time awake and/or physiologic recovery need), and cytokines IL-1 and TNF influence adenosine-mediated effects [271]. Likewise, overnight cytokine activity has been demonstrated to be altered in anxiety, including PTSD [272], indicating that abnormalities in these inflammatory factors may contribute to sleep disturbances in anxiety.

One example of an approach that targets a signaling pathway outside the intrinsic sleep or wake-promoting networks is a small trial of nabilone, a synthetic cannabinoid with agonist properties for the CB1 receptor. Cannabis, which contains hundreds of 


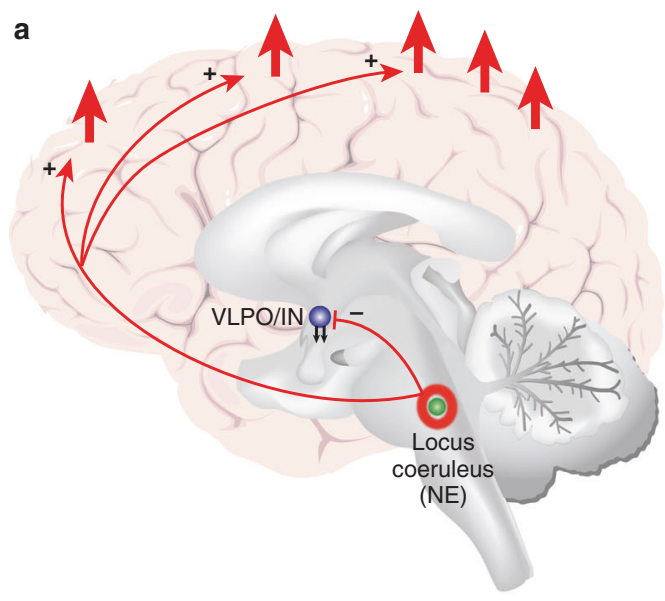

b
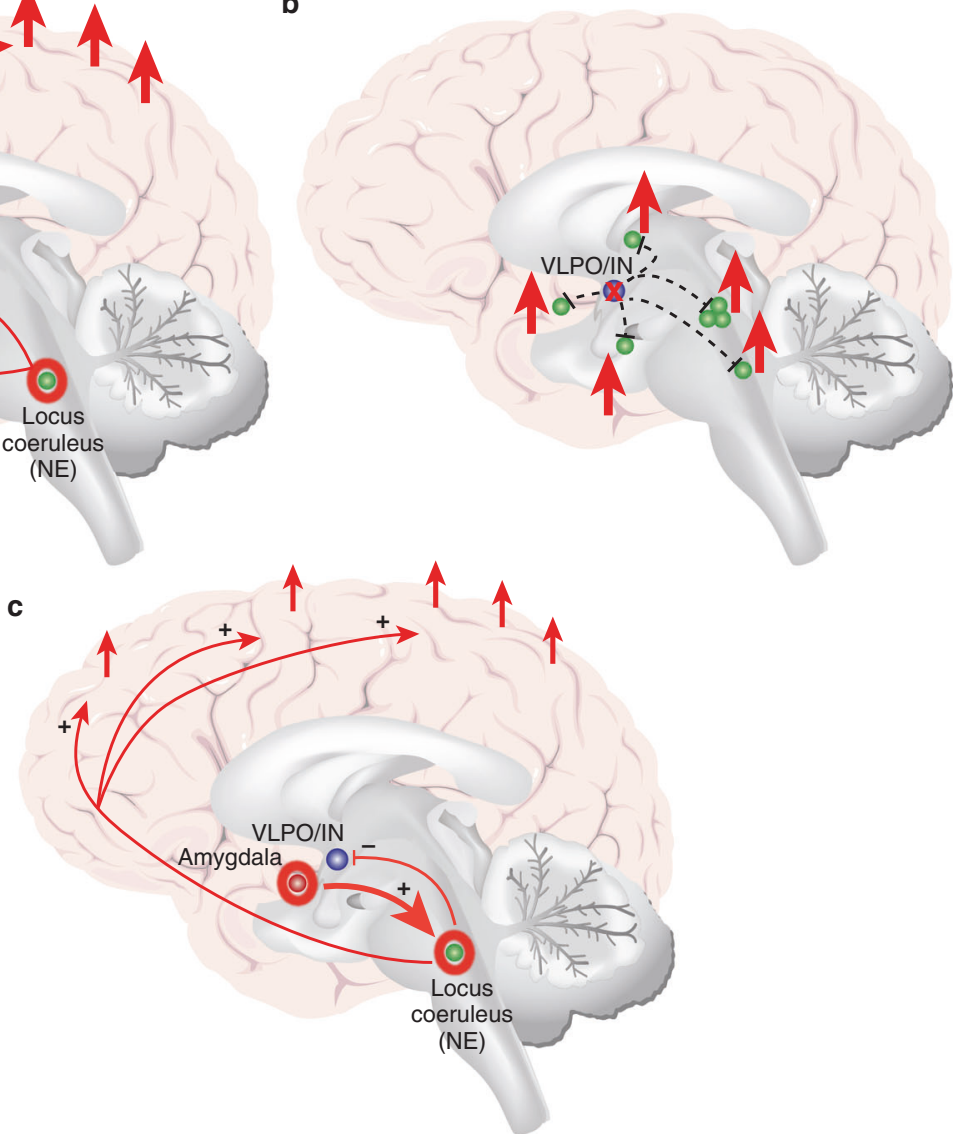

Fig. 5 Heuristic neurobiological framework for the sleep disturbance and anxiety disorder relationship. Abnormal activation in wake, NREMS, and/or anxiety regions may generate sleep disturbance in anxiety-related disorders and promote a bidirectional sleep-disturbance-anxiety relationship. a Sleep disturbance emerges from hyperactivation in intrinsic wake circuitry. For example, here, excess arousal in NE-generating LC (green with orange halo) results in inhibition of VLPO/Intermediate Nucleus (IN) (blue) and hyperactivation of cerebral cortex. b Sleep disturbance emerges from dysfuncton in central (NREM) sleep-promoting region. For example, here failure of VLPO GABA signaling (blue, with orange " $X$ ") disinhibits arousal centers (green). c Sleep disturbance emerges from hyperactivation of fear/threat/anxiety regions. For example, here amygdala hyperactivation (red, with orange halo) sends excitatory inputs to LC, which then inhibits VLPO-mediated sleep promotion and sends excitatory inputs to cerebral cortex, resulting in cortical hyperarousal, as in a. A common node in these examples is LC and NE signaling, but aberrant activity in other wake, NREMS, REMS, and anxiety regions may produce analogous effects

cannabinoids, is widely used as self-medication for anxiety and sleep problems, but the existing evidence of long-term therapeutic benefit is scarce and overall unfavorable, at least for PTSD $[273,274]$. On the other hand, cannabidiol, as opposed to tetrahydrocannabinol, shows some promise in preliminary research for sleep disturbance [273]. A trial of nabilone was predicated on evidence from PET imaging for elevated brain cannabinoid CB1 receptor availability in the amygdala of PTSD, which was associated with attentional bias to threat [275]. This small trial, which showed some efficacy for improving sleep in military-related PTSD represents a proof of concept trial of a sleep-related therapeutic targeting a pathway putatively known to be abnormal in the specific clinical population. Another potential target in the endocannabinoid system is fatty acid amide hydrolase, which metabolizes endogenous cannabinoids [276].

Summary of Important Neuromodulators in Sleep and Anxiety The above-described CNS neuromodulators have well-described roles in sleep regulation and/or anxiety, and may be targets for pharmacotherapy of sleep disturbance in anxiety-related disorders. Norepinephrine and hypocretin stand out as obvious targets that are currently under study, but novel research is pointing to a host of other neuromodulators worthy of study as potential drug targets.

A heuristic neurobiological framework for understanding sleep disturbance in anxiety disorders

Given what is known about the brain pathways (see introduction) and neuromodulators regulating sleep and anxiety, a logical assumption is that sleep disturbance and anxiety-related disorders can emanate from distinct innate or acquired neurobiological abnormalities which can then impact each other in mutually reinforcing ways [196]. Figure 5 presents a heuristic model for considering the neurobiological dynamics underlying the sleep-anxiety relationship modeled in Fig. 4. For example, the emergence of anxiety-driven sleep disturbance (i.e., anxiety first) results from the input of anxiety circuitry on sleep-wake regulatory neurocircuitry. These anxiety-related arousal pathways are likely to emanate from brain regions involved in fear and/or threat responses. The end result is disruption of NREM-generating circuitry, especially nodes most important for the generation of deep, restorative (i.e., N3/slowwave) sleep, or the over-riding of sleep-promoting signals [277]. The effects of anxiety circuitry inputs on REMS circuitry remain to be clarified, as research in humans and animals indicates 
68

disruptions as well as increases in REMS in response to stress or in PTSD subjects [120, 125, 127, 128, 278, 279].

In the other direction, abnormalities in sleep-wake regulatory circuitry in the form of heightened arousal signals or deficits in NREM sleep circuitry may impact anxiety-related neurocircuitry directly. They will also act indirectly via the cerebral cortex to generate cortical hyperarousal during wake and sleep, while at the same time impeding sleep-recovery processes that reinforce frontal-lobe-mediated functions such as emotion regulation and adaptive emotional memory processes

Finally, disruptions in neurocircuitry and modulators with fundamental roles in both sleep regulation and anxiety-related circuitry, such as NE, CRF, and hypocretin, can affect both types of disturbance. Regardless of the source cause, pathological processes emanating from either source influence each other and can become mutually reinforcing.

\section{SUMMARY, IMPORTANT UNDISCUSSED TOPICS, FUTURE DIRECTIONS}

Summarizing the above findings, the clinical and epidemiological research indicates that anxiety disorders and subjective/ clinical sleep disturbance are highly co-occurring. The objective physiological data provide a mixed picture, but overall suggest cortical hyperarousal during sleep in anxiety-related disorders, characterized by interrupted or shortened sleep, increased lighter stages of sleep (NREM N1 and N2), reductions in SWS (N3), and higher REM density (in PTSD). More-sophisticated analysis of existing data that move beyond simple sleep-stage analysis are likely necessary to better understand hyperarousal in anxiety-related sleep disturbance. The dominant psychological models of insomnia, which underscore the mutually reinforcing effects of fear and anxiety-based cognitive hyperarousal in the sleep environment and maladaptive behavioral coping, go a long way toward explaining bidirectional mechanisms driving the sleep-disturbance anxiety-disorder relationship. Insomnia models emphasizing multi-domain hyperarousal as a risk factor and endpoint in insomnia are consistent with various sleep neurobiological indicators of arousal in insomnia and are consistent with findings in anxiety-disordered sleep. Beyond insomnia models, abnormalities in cognitive inhibition, emotion regulation, and memory consolidation result from sleep disturbance and are features of anxiety disorders, and therefore have implications for bidirectional mechanisms in the anxiety-disorder-sleep-disturbance relationship. Finally, animal and human translational research as well as pharmacological treatment research provide clues to important neuromodulatory pathways that may be relevant to this relationship. A heuristic neurobiological framework indicates how sleep/wake regulatory centers may be affected during sleep in anxiety-related disorders, and suggests direct and indirect pathways through which sleep and anxiety neurobiology may affect each other.

In this review, we broadly defined anxiety-related disorders as DSM disorders in which fear and/or anxiety are considered to play a core and primary role. This reflects an attempt to maintain a faithful connection to the clinical phenomena and the human research, which has been conducted, until recently, using case/ controls approaches, while also recognizing that an approach guided by current knowledge of neurocircuitry (i.e., mediating arousal, fear and anxiety processes) in psychopathology has many advantages. The Research Domain Criteria (RDoC) encourages researchers to focus on transdiagnostic mental processes that correspond better with known neurocircuitry than do DSM disorders because they are more likely to advance our understanding of psychopathology. Therefore, rather than recruiting uniquely for a specific anxiety-related disorder (e.g., PTSD) or sleep disorder (e.g., insomnia), investigators should consider recruiting for a specific domain or symptom presentation (e.g., individuals who report high-anxiety sensitivity, individuals with poor sleep continuity). The RDoC also emphasizes the importance of integrating many levels of information (e.g., genetics, neurobiological data from human and animal subjects, subjective-report data). In line with this model, future studies will benefit from multimodal assessments in order to better understand the intricacies of relevant mechanisms.

Although reviewing this vast topic, it is important to acknowledge items not covered in this review that are important when considering sleep and anxiety-related disorders. First, the effects of circadian rhythm and circadian disruption on sleep and anxietyrelated disorders are outside of the scope of this review, but are fundamental contributors to the disturbances in question. For example, there is evidence that circadian factors impact anxiety symptom expression, extinction memory, and exposure therapy efficacy, although discrimination between circadian and homeostatic contributors is not clear in most studies [280, 281]. We refer readers to existing studies which address this topic [83, 152, 282287] and encourage the consideration of circadian factors in sleep research, and anxiety research, in general. In addition, we have focused on anxiety disorders, but the comorbidity and overlap with mood disorders is considerable [288, 289]. The effects reviewed in this report often, but not always, accounted for the effects of mood disorders such as major depressive disorder. Finally, the genetics underlying the above-described relationships are outside of the scope of the current review but genetics research is essential for understanding shared and overlapping etiologies.

The future of therapeutic progress in improving outcomes for individuals with sleep disturbance in anxiety-related disorders will involve expanding upon existing excellent cognitive-behavioral treatments and integrating these with tools developed based on a deeper grasp of neurobiology. Dias and colleagues [290] predict that advanced technologies that target specific disturbed pathways in the human body, in the way that optogenetic manipulations already affect anxiety in animal models, are on the horizon. Combining the least invasive biological methods with the many advantages of psychological treatments should be the objective of medical science in sleep and anxiety-related disorders into the future.

\section{FUNDING AND DISCLOSURE}

Dr. Neylan is a consultant for Jazz Pharmaceuticals. Dr. Kanady and Dr. Richards declare nopotential conflicts of interest. The authors declare no competing interests.

\section{ADDITIONAL INFORMATION}

Publisher's note: Springer Nature remains neutral with regard to jurisdictional claims in published maps and institutional affiliations.

\section{REFERENCES}

1. Van Someren, EJW et al. Disrupted sleep: from molecules to cognition. J Neurosci. 2015;35:13889-95.

2. Goel N, Rao H, Durmer JS, Dinges DF. Neurocognitive consequences of sleep deprivation. Semin Neurol. 2009;29:320-339.

3. Killgore WDS. Effects of sleep deprivation on cognition. Prog Brain Res. 2010;185:105-29.

4. Gruber R, Cassoff J. The interplay between sleep and emotion regulation: conceptual framework empirical evidence and future directions. Curr Psychiatry Rep. 2014;16:500

5. Reutrakul S, Van Cauter E. Sleep influences on obesity, insulin resistance, and risk of type 2 diabetes. Metabolism. 2018;84:56-66.

6. Ibarra-Coronado EG, et al. The Bidirectional Relationship between Sleep and Immunity against Infections. J Immunol Res. 2015;2015:678164.

7. Aserinsky E, Kleitman N. Regularly occurring periods of eye motility, and concomitant phenomena, during sleep. Science. 1953;118:273-74 
8. Dement W, Kleitman N. Cyclic variations in EEG during sleep and their relation to eye movements, body motility, and dreaming. Electroencephalogr Clin Neurophysiol. 1957;9:673-90

9. Jouvet M. The States of Sleep. Sci Am. 1967;216:62-8.

10. Tononi G, Cirelli C. Sleep and the price of plasticity: from synaptic and cellular homeostasis to memory consolidation and integration. Neuron. 2014;81:12-34.

11. Tononi G, Cirelli C. Sleep function and synaptic homeostasis. Sleep Med Rev. 2006;10:49-62.

12. Richards $A$, et al. Sleep and cognitive performance from teens to old age: more is not better. Sleep. 2017;40: https://doi.org/10.1093/sleep/zsw029.

13. Pace-Schott EF, Germain A, Milad MR. Effects of sleep on memory for conditioned fear and fear extinction. Psychol Bull. 2015; https://doi.org/10.1037/ bul0000014.

14. Groch S, Wilhelm I, Diekelmann S, Born J. The role of REM sleep in the processing of emotional memories: Evidence from behavior and event-related potentials. Neurobiol Learn Mem. 2013; https://doi.org/10.1016/j.nlm.2012.10.006.

15. Groch S, Zinke K, Wilhelm I, Born J. Dissociating the contributions of slow-wave sleep and rapid eye movement sleep to emotional item and source memory. Neurobiol Learn Mem. 2015; https://doi.org/10.1016/j.nlm.2014.08.013.

16. Czeisler CA, et al. Stability, precision, and near-24-hour period of the human circadian pacemaker. Science. 1999;284:2177-81.

17. Borbely AA. A two process model of sleep regulation. Hum Neurobiol 1982;1:195-204

18. Oh J. et al. The role of co-neurotransmitters in sleep and wake regulation. Mol Psychiatry. 2018; https://doi.org/10.1038/s41380-018-0291-2.

19. Schwartz MD, Kilduff TS. The neurobiology of sleep and wakefulness. Psychiatr Clin North Am. 2015; https://doi.org/10.1016/j.psc.2015.07.002.

20. Saper CB, Scammell TE, Lu J. Hypothalamic regulation of sleep and circadian rhythms. Nature. 2005;437:1257-63.

21. Behn CGD, Brown EN, Scammell TE, Kopell NJ. Mathematical model of network dynamics governing mouse sleep-wake behavior. J Neurophysiol 2007;97:3828-40.

22. Phillips AJK, Robinson PA. A quantitative model of sleep-wake dynamics based on the physiology of the brainstem ascending arousal system. J Biol Rhythms. 2007;22:167-79.

23. Rempe MJ, Best J, Terman D. A mathematical model of the sleep/wake cycle. J Math Biol 2010;60:615-44.

24. Saper CB, Fuller PM. wake-sleep circuitry: an overview. Curr Opin Neurobiol 2017;44:186-92.

25 . Anaclet $C$, et al. Identification and characterization of a sleep-active cell group in the rostral medullary brainstem. J Neurosci 2012;32:17970-6.

26. Anaclet $C$, et al. The GABAergic parafacial zone is a medullary slow wave sleeppromoting center. Nat Neurosci 2014;17:1217-24.

27. Saper CB, Fuller PM, Pedersen NP, Lu J, Scammell TE. Sleep State Switching. Neuron. 2010; https://doi.org/10.1016/j.neuron.2010.11.032.

28. Nofzinger EA, et al. Functional neuroimaging evidence for hyperarousal in insomnia. Am. J. Psychiatry. 2004; https://doi.org/10.1176/appi.ajp.161.11.2126.

29. Germain A, Stocker R, Ebdlahad S, Mammen O. Functional neuroimaging of NREM sleep in combat veterans with and without posttraumatic stress disorder (PTSD). Sleep. 2013;211:176-79.

30. Ebdlahad S, Stocker R, Mammen O, Nofzinger E, Germain A. Neural correlates of insomnia comorbid with PTSD during wakefulness and nrem sleep. Sleep. 2013;36:A303.

31. Etkin A, Gyurak A, O'Hara, R. A neurobiological approach to the cognitive deficits of psychiatric disorders. Dialogues Clin. Neurosci. 2013;15: 419-429.

32. Etkin A, Wager TD. Functional neuroimaging of anxiety: A meta-ana lysis of emotional processing in PTSD, social anxiety disorder, and specific phobia. Am J Psychiatry. 2007; https://doi.org/10.1176/appi.ajp.2007.07030504.

33. Shin LM, Liberzon I. The neurocircuitry of fear, stress, and anxiety disorders. Neuropsychopharmacology. 2010; https://doi.org/10.1038/npp.2009.83.

34. Duval ER, Javanbakht A, Liberzon I. Neural circuits in anxiety and stress disorders: a focused review. Ther Clin Risk Manag. 2015; https://doi.org/10.2147/ TCRM.S48528.

35. Norton PJ, Paulus DJ. Transdiagnostic models of anxiety disorder: theoretica and empirical underpinnings. Clin Psychol Rev 2017;56:122-37.

36. Neylan TC, et al. Sleep disturbances in the Vietnam generation: findings from a nationally representative sample of male Vietnam Veterans. Am J Psychiatry. 1998;155:929-33.

37. Monti JM, Monti D. Sleep disturbance in generalized anxiety disorder and its treatment. Sleep Med Rev 2000:4:263-76.

38. Stewart R, et al. Insomnia comorbidity and impact and hypnotic use by age group in a national survey population aged 16 to 74 years. Sleep. 2006;29: 1391-7.
39. Leskin GA, Woodward SH, Young HE, Sheikh Jl. Effects of comorbid diagnoses on sleep disturbance in PTSD. J Psychiatr Res 2002;36:449-52.

40. Ramsawh HJ, Stein MB, Belik SL, Jacobi F, Sareen J. Relationship of anxiety disorders, sleep quality, and functional impairment in a community sample. J Psychiatr Res 2009;43:926-33.

41. Cox RC, Olatunji BO. A systematic review of sleep disturbance in anxiety and related disorders. J Anxiety Disord 2016;37:104-29.

42. Germain A. Sleep disturbances as the hallmark of PTSD: where are we now? Am J Psychiatry. 2013;170:372-82.

43. Ross RJ, Ball WA, Sullivan KA, Caroff SN. Sleep disturbance as the Hallmark of posttraumatic stress disorder. Am J Psychiatry. 1989;146:697-707.

44. Roszell DK, McFall ME, Malas KL. Frequency of symptoms and concurrent psychiatric disorder in Vietnam veterans with chronic PTSD. Hosp Community Psychiatry. 1991;42:293-6.

45. Ohayon MM, Shapiro CM. Sleep disturbances and psychiatric disorders associated with posttraumatic stress disorder in the general population. Compr Psychiatry. 2000;41:469-78.

46. American Psychiatric Association. Diagnostic and statistical manual of mental disorders. Am Psychiatr Assoc. 2013; https://doi.org/10.1176/appi. books. 9780890425596.744053

47. Creamer JL, Brock MS, Mysliwiec V. Nightmares in United States military personnel are multifactorial and require further astudy. J Clin Sleep Med 2018;14:1275-6.

48. Sandman N, et al. Nightmares: prevalence among the Finnish general adult population and war veterans during 1972-2007. Sleep. 2013;36:1041-50.

49. Bixler EO, Kales A, Soldatos CR, Kales JD, Healey S. Prevalence of sleep disorders in the Los Angeles metropolitan area. Am J Psychiatry. 1979;136:1257-62.

50. Bjorvatn B, Grønli J, Pallesen S. Prevalence of different parasomnias in the general population. Sleep Med 2010;11:1031-4.

51. Li SX, Zhang B, Li AM, Wing YK. Prevalence and correlates of frequent nightmares: a community-based 2-phase study. Sleep. 2010;33:774-80.

52. Robert G, Zadra A. Measuring nightmare and bad dream frequency: Impact of retrospective and prospective instruments. J Sleep Res 2008;17:132-9.

53. Kobayashi I, Delahanty DL. Gender differences in subjective sleep after trauma and the development of posttraumatic stress disorder symptoms: sa pilot study. J Trauma Stress. 2013;26:467-74.

54. Milanak ME, et al. Traumatic event exposure, posttraumatic stress disorder, and sleep disturbances in a national sample of U.S. adults. J Trauma Stress. 2019;32:14-22.

55. Woodward SH, Arsenault NJ, Murray C, Bliwise DL. Laboratory sleep correlates of nightmare complaint in PTSD inpatients. Biol Psychiatry. 2000;48:1081-7.

56. Insana SP, Hall M, Buysse DJ, Germain A. Validation of the pittsburgh sleep quality index addendum for posttraumatic stress disorder (PSQI-A) in U.S. male military veterans. J Trauma Stress. 2013;26:192-200.

57. Mysliwiec V, et al. Trauma associated sleep disorder: a parasomnia induced by trauma. Sleep Med Rev 2018;37:94-104.

58. Brown TM, Boudewyns PA. Periodic limb movements of sleep in combat veterans with posttraumatic stress disorder. J Trauma Stress. 1996;9:129-36.

59. Mellman TA, Kulick-Bell R, Ashlock LE, Nolan B. Sleep events among veterans with combat-related posttraumatic stress disorder. Am J Psychiatry. 1995:152:110-5.

60. Ross RJ, et al. Motor dysfunction during sleep in posttraumatic stress disorder Sleep. 1994;17:723-32.

61. Woodward SH, Michell G, Santerre C. The psychophysiology of PTSD nightmares. in Sleep and Combat-Related Post Traumatic Stress Disorder (2017). https://doi.org/10.1007/978-1-4939-7148-0_20.

62. Mysliwiec V, et al. Trauma associated sleep disorder: sa proposed parasomnia encompassing disruptive nocturnal behaviors, nightmares, and REM without atonia in trauma survivors. J Clin Sleep Med 2014;10:1143-8.

63. Bélanger L, Morin CM, Langlois F, Ladouceur R. Insomnia and generalized anxiety disorder: effects of cognitive behavior therapy for gad on insomnia symptoms. J Anxiety Disord 2004;18:561-71.

64. Papadimitriou GN, Linkowski P. Sleep disturbance in anxiety disorders. Int Rev Psychiatry. 2005;17:229-36.

65. Brenes GA, et al. Insomnia in older adults with generalized anxiety disorder. Am J Geriatr Psychiatry. 2009;17:465-72.

66. Tempesta D, et al. Neuropsychological functioning in young subjects with generalized anxiety disorder with and without pharmacotherapy. Prog NeuroPsychopharmacol Biol Psychiatry. 2013;45:236-41.

67. Wetherell JL, Le Roux H, Gatz M. DSM-IV criteria for generalized anxiety disorder in older adults: distinguishing the worried from the well. Psychol Aging 2003;18:622-7.

68. Berger $A$, et al. Patterns of healthcare utilization in patients with generalized anxiety disorder in general practice in Germany. Eur J Psychiat. 2009; 23:90-100. 
69. Mellman TA, Uhde TW. Patients with frequent sleep panic: clinical findings and response to medication treatment. J Clin Psychiatry. 1990;51:513-26.

70. Park HJ, et al. Association between sleep disorder and panic disorder in South Korea: nationwide nested case-control study of data from 2004 to 2013. Psychiatry Res 2018;260:286-91.

71. Sheehan DV, Ballenger J, Jacobsen G. Treatment of endogenous anxiety with phobic, hysterical, and hypochondriacal symptoms. Arch Gen Psychiatry. 1980;37:51-59.

72. Overbeek T, Van Diest R, Schruers K, Kruizinga F, Griez E. Sleep complaints in panic disorder patients. J Nerv Ment Dis 2005;193:488-93.

73. Todder D, Baune BT. Quality of sleep in escitalopram-treated female patients with panic disorder. Hum Psychopharmacol. 2010;25:167-73.

74. Craske MG, Tsao JC. Assessment and treatment of nocturnal panic attacks. Sleep Med Rev 2005;9:173-84.

75. Castelnovo A, Lopez R, Proserpio P, Nobili L, Dauvilliers Y. NREM sleep parasomnias as disorders of sleep-state dissociation. Nat Rev Neurol. 2018; https:// doi.org/10.1038/s41582-018-0030-y.

76. Craske MG, Barlow DH. Nocturnal panic. J Nerv Ment Dis 1989;173:160-7.

77. Krystal JH, Woods SW, Hill CL, Charney DS. Characteristics of panic attack subtypes: sassessment of spontaneous panic, situational panic, sleep panic, and limited symptom attacks. Compr Psychiatry. 1991;32:474-80.

78. Stein MB, Enns MW, Kryger MH. Sleep in nondepressed patients with panic disorder: II. Polysomnographic assessment of sleep architecture and sleep continuity. J Affect Disord 1993;28:1-6.

79. Uhde TW, Anxiety Disorders. in Principles and Practice of Sleep Medicine,. Kryger Meir H; Roth, TDWC (ed.) 871-98 (Saunders Co., 1994).

80. Mellman TA, Uhde TW. Electroencephalographic sleep in panic disorder: a focus on sleep-related panic attacks. Arch Gen Psychiatry. 1989;46:178-84.

81. Ağargün MY, Kara H. Recurrent sleep panic, insomnia, and suicidal behavior in patients with panic disorder. Compr Psychiatry. 1998;39:149-51.

82. Roth $\mathrm{T}$, et al. Sleep problems, comorbid mental disorders, and role functioning in the national comorbidity survey replication. Biol Psychiatry. 2006;60:1364-71.

83. Bobdey M, Fineberg N, Gale TM, Patel A, Davies HA. Reported sleep patterns in obsessive compulsive disorder (OCD). Int J Psychiatry Clin Pract 2002;6:15-21.

84. Donse L, Sack AT, Fitzgerald PB, Arns M. Sleep disturbances in obsessivecompulsive disorder: association with non-response to repetitive transcranial magnetic stimulation (rTMS). J Anxiety Disord. 2017; https://doi.org/10.1016/j. janxdis.2017.03.006

85. Park S, et al. Relationships of sleep duration with sociodemographic and healthrelated factors, psychiatric disorders and sleep disturbances in a community sample of Korean adults. J Sleep Res 2010;19:567-77.

86. Robinson D, Walsleben J, Pollack S, Lerner G. Nocturnal polysomnography in obsessive-compulsive disorder. Psychiatry Res 1998;80:257-63.

87. Marcks BA, Weisberg RB, Edelen MO, Keller MB. The relationship between sleep disturbance and the course of anxiety disorders in primary care patients. Psychiatry Res 2010;178:487-92.

88. Daroff RB. The International Classification of Sleep Disorders: Diagnostic and Coding Manual. Neurology. 2012; https://doi.org/10.1212/wnl.41.1.160.

89. Eckert DJ, Malhotra A. Pathophysiology of adult obstructive sleep apnea. Proc Am Thorac Soc. 2008; https://doi.org/10.1513/pats.200707-114mg.

90. Younes M. Pathogenesis of obstructive sleep Apnea. Clin Chest Med. 2019; https://doi.org/10.1016/j.ccm.2019.02.008.

91. Colvonen PJ, et al. Obstructive sleep apnea and posttraumatic stress disorder among OEF/OIF/OND veterans. J Clin Sleep Med 2015;11:513-8.

92. Yesavage JA, et al. Sleep-disordered breathing in vietnam veterans with posttraumatic stress disorder. Am J Geriatr Psychiatry. 2012;20:199-204.

93. Krakow B, et al. Sleep-disordered breathing, psychiatric distress, and quality of life impairment in sexual assault survivors. J Nerv Ment Dis 2002;190:442-52.

94. Krakow B, et al. Complex insomnia: Insomnia and sleep-disordered breathing in a consecutive series of crime victims with nightmares and PTSD. Biol Psychiatry. 2001;49:948-53.

95. Ocasio-Tascón ME, Alicea-Colón E, Torres-Palacios A, Rodríguez-Cintrón W. The veteran population: one at high risk for sleep-disordered breathing. Sleep Breath 2006;10:70-75.

96. Spoormaker VI, Montgomery P. Disturbed sleep in post-traumatic stress disorder: saecondary symptom or core feature? Sleep Med Rev 2008;12:169-84.

97. Youakim JM, Doghramji K, Schutte SL. Posttraumatic stress disorder and obstructive sleep apnea syndrome. Psychosomatics. 1998:39:168-71.

98. Krakow BJ, Ulibarri VA, Moore BA, Mclver ND. Posttraumatic stress disorder and sleep-disordered breathing: a review of comorbidity research. Sleep Med Rev. 2015; https://doi.org/10.1016/j.smrv.2014.11.001.

99. Engdahl BE, Eberly RE, Hurwitz TD, Mahowald MW, Blake J. Sleep in a community sample of elderly war veterans with and without posttraumatic stress disorder. Biol Psychiatry. 2000;47:520-5.
100. Kinoshita LM, et al. Modeling the effects of obstructive sleep apnea and hypertension in Vietnam veterans with PTSD. Sleep Breath 2012;16:1201-9.

101. Gupta MA, Simpson FC. Obstructive sleep apnea and psychiatric disorders: a systematic review. J Clin Sleep Med 2015;11:165-75.

102. Mysliwiec V, et al. Sleep disorders and sassociated medical comorbidities in sactive duty military personnel. Sleep. 2013;36:167-74.

103. Mysliwiec V, et al. Sleep disorders in US military personnel: a high rate of comorbid insomnia and obstructive sleep apnea. Chest. 2013;144:549-57.

104. van Liempt, S, Westenberg, HG, Arends, J, Vermetten, E. Obstructive sleep apnea in combat-related posttraumatic stress disorder: a controlled polysomnography study. Eur J Psychotraumatol 2011;2:10.3402/ejpt.v2i0.8451.

105. Zhang Y, Weed JG, Ren R, Tang X, Zhang W. Prevalence of obstructive sleep apnea in patients with posttraumatic stress disorder and its impact on adherence to continuous positive airway pressure therapy: a meta-analysis. Sleep Med 2017;36:125-32.

106. Babson KA, Del Re AC, Bonn-Miller MO, Woodward SH. The comorbidity of sleep apnea and mood, anxiety, and substance use disorders among obese military veterans within the veterans health administration. J Clin Sleep Med 2013;9:1253-8.

107. Pevernagie $D$, et al. Behavioural hyperventilation as a novel clinical condition associated with central sleep apnoea: a report of three cases. Sleep Med 2012;13:1317-20

108. Trajanovic NN, Rasool MS, Voloh I, Shapiro CM. Sleep-disordered breathing, cardiac arrhythmia, and panic disorder. J Clin Psychol Med Settings. $2005 ; 1: 288-9$

109. Enns MW, Stein M, Kryger M. Successful treatment of comorbid panic disorder and sleep apnea with continuous positive airway pressure. Psychosomatics. 1995;36:585-6.

110. Stein MB, Millar TW, Larsen DK, Kryger MH. Irregular breathing during sleep in patients with panic disorder. Am J Psychiatry. 1995;152:1168-73.

111. Edlund MJ, McNamara ME, Millman RP. Sleep apnea and panic attacks. Compr Psychiatry. 1991;32:130-2.

112. Hrubos-Strøm $\mathrm{H}$, et al. Sleep apnoea, anxiety, depression and somatoform pain: sa community-based high-risk sample. Eur Respir J 2012;40:400-7.

113. Breland JY, et al. The obesity epidemic in the veterans health administration: prevalence samong key populations of women and men veterans. J Gen Intern Med. 2017; https://doi.org/10.1007/s11606-016-3962-1.

114. Rosenbaum $S$, et al. The prevalence and risk of metabolic syndrome and its components among people with posttraumatic stress disorder: a systematic review and meta-analysis. Metabolism 2015;64:926-33.

115. Baglioni $C$, et al. Sleep and mental disorders: sa meta-analysis of polysomnographic research. Psychol Bull 2016;142:969-90.

116. Kobayashi I, Boarts JM, Delahanty DL. Polysomnographically measured sleep abnormalities in PTSD: a meta-analytic review. Psychophysiology. 2007;44:660-9.

117. Koren D, Arnon I, Lavie P, Klein E. Sleep complaints as early predictors of posttraumatic stress disorder: a 1-year prospective study of injured survivors of motor vehicle accidents. Am J Psychiatry. 2002;159:855-7.

118. Fuller $\mathrm{KH}$, Waters WF, Scott O. An investigation of slow-wave sleep processes in chronic PTSD patients. J Anxiety Disord 1994;8:227-36.

119. Mellman TA, Nolan B, Hebding J, Kulick-Bell R, Dominguez R. A polysomnographic comparison of veterans with combat-related PTSD, depressed men, and non-ill controls. Sleep. 1997;20:46-51.

120. Richards A, et al. Sex differences in objective measures of sleep in posttraumatic stress disorder and healthy control subjects. J Sleep Res 2013;22:679-87.

121. Habukawa M, Uchimura N, Maeda M, Kotorii N, Maeda $H$. Sleep findings in young adult patients with posttraumatic stress disorder. Biol Psychiatry. 2007;62:1179-82.

122. Breslau N, et al. Sleep in lifetime posttraumatic stress disorder: a communitybased polysomnographic study. Arch Gen Psychiatry. 2004;61:508-16.

123. Mellman TA, Bustamante V, Fins Al, Pigeon WR, Nolan B. REM sleep and the early development of posttraumatic stress disorder. Am J Psychiatry. 2002; https:// doi.org/10.1176/appi.ajp.159.10.1696.

124. Mellman TA, Kobayashi I, Lavela J, Wilson B, Hall Brown TS. A relationship between REM sleep measures and the duration of posttraumatic stress disorder in a young adult urban minority population. Sleep. 2014;1:1321-6.

125. Ross RJ. The changing REM sleep signature of posttraumatic satress disorder. Sleep. 2014;37:1281-2.

126. Sanford LD, Suchecki D, Meerlo P. Stress, arousal, and sleep. Curr Top Behav Neurosci. 2015; https://doi.org/10.1007/7854 2014314.

127. Wellman LL, Yang L, Ambrozewicz MA, Machida M, Sanford LD. Basolateral amygdala and the regulation of fear-conditioned changes in sleep: role of corticotropin-releasing factor. Sleep. 2013; https://doi.org/10.5665/sleep.2526. 
128. Vanderheyden WM, et al. Sleep alterations following exposure to stress predict fear-associated memory impairments in a rodent model of PTSD. Exp Brain Res 2015;233:2335-46.

129. Vanderheyden W, Urpa L, Poe G. Increase in rem sleep following trauma exposure. Sleep Med. 2014; https://doi.org/10.1016/j.sleep.2013.11.718.

130. Suchecki D, Tiba PA, Machado RB. REM sleep rebound as an adaptive response to stressful situations. Front Neurol. 2012; https://doi.org/10.3389/ fneur.2012.00041.

131. Pawlyk AC, Morrison AR, Ross RJ, Brennan FX. Stress-induced changes in sleep in rodents: models and mechanisms. Neurosci Biobehav Rev. 2008; https://doi.org/ 10.1016/j.neubiorev.2007.06.001.

132. Germain A, James JA, Mammen O, Price J, Nofzinger E. Functional neuroimaging of rem sleep in returning veterans with ptsd: san [18F]-FDG PET study. Sleep. 2011;A242:34

133. Suter D, Mammen O, Insana S, Nofzinger E, Germain A. Neurobiological effects of prazosin on NREM sleep in Veterans with PTSD. in SLEEP - Abstract Supplement, Volume 37 (2014)

134. Germain A, et al. Prazosin increases brain glucose metabolism in regions involved in fear extinction learning and memory during REM sleep in combat exposed Veterans with PTSD. in SLEEP - Abstract Supplement, Volume 37 (2014).

135. Van Wyk M, Thomas KGF, Solms M, Lipinska G. Prominence of hyperarousal symptoms explains variability of sleep disruption in posttraumatic stress disorder. Psychol Trauma. 2016;8:688-96.

136. Mellman TA, Kumar A, Kulick-Bell R, Kumar M, Nolan B. Nocturnal/daytime urine noradrenergic measures and sleep in combat-related PTSD. Biol Psychiatry. 1995;38:174-9.

137. Inslicht SS, et al. Sleep and hypothalamic pituitary adrenal axis responses to metyrapone in posttraumatic stress disorder. Psychoneuroendocrinology. 2018;88:136-43.

138. Van Liempt $\mathrm{S}$, et al. Sympathetic activity and hypothalamo-pituitary-adrenal axis activity during sleep in post-traumatic stress disorder: a study assessing polysomnography with simultaneous blood sampling. Psychoneuroendocrinology. 2013;38:155-65.

139. Papadimitriou GN, Kerkhofs M, Kempenaers C, Mendlewicz J. EEG sleep studies in patients with generalized anxiety disorder. Psychiatry Res 1988;26:183-90.

140. Arriaga F, Paiva T. Clinical and EEG sleep changes in primary dysthymia and generalized anxiety: a comparison with normal controls. Neuropsychobiology. 1990;24:109-14.

141. Lund HG, Bech $P$, Eplov L, Jennum P, Wildschiødtz G. An epidemiological study of REM latency and psychiatric disorders. J Affect Disord 1991;23:107-12.

142. Lauer CJ, Krieg JC, Garcia-Borreguero D, Özdaglar A, Holsboer F. Panic disorder and major depression: a comparative electroencephalographic sleep study. Psychiatry Res 1992;44:41-54.

143. Lydiard RB, et al. Electroencephalography during sleep of patients with panic disorder. J Neuropsychiatry Clin Neurosci 1989;1:372-6.

144. Sloan EP, et al. Nocturnal and daytime panic attacks comparison of sleep architecture, heart rate variability, and response to sodium lactate challenge. Biol Psychiatry. 1999;45:1313-20.

145. Uhde TW, et al. The sleep of patients with panic disorder: sa preliminary report. Psychiatry Res 1984;12:251-9.

146. Ferini-Strambi L, et al. Cyclic alternating pattern of sleep electroencephalogram in patients with panic disorder. Biol Psychiatry. 1996;40:225-6.

147. Dubé $S$, et al. Interface of panic and depression: clinical and sleep EEG correlates. Psychiatry Res 1986;19:119-33.

148. Insel TR, et al. The sleep of patients with obsessive-compulsive disorder. Arch Gen Psychiatry. 1982;39:1372-7.

149. Voderholzer U, et al. Sleep in obsessive compulsive disorder: Polysomnographic studies under baseline conditions and after experimentally induced serotonin deficiency. Eur Arch Psychiatry Clin Neurosci 2007;257:173-82.

150. Hohagen F, et al. Sleep EEG of patients with obsessive-compulsive disorder. Eur Arch Psychiatry Clin Neurosci 1994;243:273-8

151. Kluge $M$, et al. Increased nocturnal secretion of ACTH and cortisol in obsessive compulsive disorder. J Psychiatr Res 2007;41:928-33.

152. Nota JA, Sharkey KM, Coles ME. Sleep, arousal, and circadian rhythms in adults with obsessive-compulsive disorder: sa meta-analysis. Neurosci Biobehav Rev.2015; https://doi.org/10.1016/j.neubiorev.2015.01.002.

153. Otte $C$, et al. Effects of Metyrapone on hypothalamic-pituitary-adrenal axis and sleep in women with post-traumatic stress disorder. Biol Psychiatry. 2007, https://doi.org/10.1016/j.biopsych.2006.08.018.

154. Woodward SH, Murburg MM, Bliwise DL. PTSD-related hyperarousal assessed during sleep. Physiol Behav. 2000, https://doi.org/10.1016/S0031-9384(00) 00271-7.

155. Wei $Y$, et al. Sleep stage transition dynamics reveal specific stage 2 vulnerability in insomnia. Sleep 2017; https://doi.org/10.1093/sleep/zsx117.
156. Nofzinger EA, et al. A method for the assessment of the functional neuroanatomy of human sleep using FDG PET. Brain Res Protoc. 1998; https://doi.org/ 10.1016/S1385-299X(97)00042-1.

157. Gehrman P, et al. Predeployment sleep duration and insomnia symptoms as risk factors for new-onset mental health disorders following military deployment. Sleep. 2013;36:1009-18.

158. Wright KM, et al. Insomnia as predictor versus outcome of PTSD and depression among Iraq combat veterans. J Clin Psychol 2011;67:1240-58.

159. Van Liempt $S$, Van Zuiden $M$, Westenberg $H$, Super A, Vermetten E. Impact of impaired sleep on the development of PTSD symptoms in combat veterans: sa prospective longitudinal cohort study. Depress Anxiety. 2013; https://doi.org/ 10.1002/da.22054

160. Talbot LS, et al. Cognitive behavioral therapy for insomnia in posttraumatic satress disorder: a randomized controlled trial. Sleep. 2014;37:327-41.

161. Ho FYY, Chan CS, Tang KNS. Cognitive-behavioral therapy for sleep disturbances in treating posttraumatic stress disorder symptoms: a meta-analysis of randomized controlled trials. Clin Psychol Rev. 2016; https://doi.org/10.1016/j. cpr.2015.09.005.

162. Hertenstein $E$, et al. Insomnia as a predictor of mental disorders: sa systematic review and meta-analysis. Sleep Med Rev. 2019;. https://doi.org/10.1016/j. smrv.2018.10.006.

163. Short NA, Allan NP, Stentz L, Portero AK, Schmidt NB. Predictors of insomnia symptoms and nightmares among individuals with post-traumatic stress disorder: san ecological momentary assessment study. J Sleep Res 2017;27:64-72.

164. Johnson EO, Roth T, Breslau N. The association of insomnia with anxiety disorders and depression: exploration of the direction of risk. J Psychiatr Res. 2006; https://doi.org/10.1016/j.jpsychires.2006.07.008.

165. Batterham PJ, Glozier N, Christensen H. Sleep disturbance, personality and the onset of depression and anxiety: prospective cohort study. Aust N Z J Psychiatry. 2012:46:1089-98.

166. Roy Byrne PP, Uhde TW, Post RM. Effects of one night's sleep deprivation on mood and behavior in panic disorder: patients with panic disorder compared with depressed patients and normal controls. Arch Gen Psychiatry. 1986:43:895-9.

167. Babson KA, Feldner MT, Trainor CD, Smith RC. An experimental investigation of the effects of acute sleep deprivation on panic-relevant biological challenge responding. Behav Ther 2009;40:239-50.

168. Cousineau $\mathrm{H}$, et al. Insomnia symptoms following treatment for comorbid panic disorder with agoraphobia and generalized anxiety disorder. J Nerv Ment Dis 2016;204:267-73.

169. Cervena K, Matousek M, Prasko J, Brunovsky M, Paskova B. Sleep disturbances in patients treated for panic disorder. Sleep Med 2005;6:149-53.

170. Karlin BE, Trockel M, Spira AP, Taylor CB, Manber R. National evaluation of the effectiveness of cognitive behavioral therapy for insomnia among older versus younger veterans. Int J Geriatr Psychiatry. 2015; https://doi.org/10.1002/ gps.4143.

171. Qaseem A, Kansagara D, Forciea MA, Cooke M, Denberg TD. Management of chronic insomnia disorder in adults: a clinical practice guideline from the American college of physicians. Ann Intern Med 2016;165:125-33.

172. Belleville G, Cousineau H, Levrier K, St-Pierre-Delorme M-E. Meta-analytic review of the impact of cognitive-behavior therapy for insomnia on concomitant anxiety. Clin Psychol Rev 2011; https://doi.org/10.1016/j.cpr.2011.02.004.

173. Spielman AJ, Caruso LS, Glovinsky PB. A behavioral perspective on insomnia treatment. Psychiatr Clin North Am 1987;10:541-53.

174. Bootzin R. Stimulus control treatment for insomnia. Proc Am Psychol Assoc 1972;7:395-6.

175. Harvey AG. A cognitive model of insomnia. Behav Res Ther 2002;40:869-93.

176. Espie CA. Insomnia: conceptual issues in the development, persistence, and treatment of sleep disorder in adults. Annu Rev Psychol 2002;53:215-43.

177. Riemann $D$ et al. The hyperarousal model of insomnia: sa review of the concept and its evidence. Sleep Med Rev. 2010; https://doi.org/10.1016/j. smrv.2009.04.002.

178. Perlis ML, Smith MT, Pigeon WR. Etiology and Pathophysiology of Insomnia. in Principles and Practice of Sleep Medicine, 714-25 (Elsevier Inc., 2005). https:// doi.org/10.1016/B0-72-160797-7/50067-7.

179. Pigeon WR. Diagnosis, prevalence, pathways, consequences, treatment of insomnia. Indian J Med Res 2010;131:321-32.

180. Sinha SS. Trauma-induced insomnia: sa novel model for trauma and sleep research. Sleep Med Rev 2016;25:74-83.

181. Lima SL, Rattenborg NC, Lesku JA, Amlaner CJ. Sleeping under the risk of predation. Anim Behav 2005;70:723-36.

182. Eban-Rothschild A, Giardino WJ, de Lecea L. To sleep or not to sleep: neuronal and ecological insights. Curr Opin Neurobiol 2017;44:132-8. 
183. Kanady JC, et al. Cognitive behavioral therapy for insomnia reduces fear of sleep in individuals with posttraumatic stress disorder. J Clin Sleep Med 2018;14:1193-203.

184. Krakow B, et al. An open-label trial of evidence-based cognitive behavior therapy for nightmares and insomnia in crime victims with PTSD. Am J Psychiatry. 2001;158:2043-7.

185. Pruiksma KE, et al. A psychometric study of the Fear of Sleep Inventory-Short Form (FoSI-SF). J Clin Sleep Med 2014;10:551-8.

186. Hall Brown T, Mellman TA. The influence of PTSD, sleep fears, and neighborhood stress on insomnia and short sleep duration in urban, young adult, african americans. Behav Sleep Med 2014;12:198-206.

187. Palagini L, Moretto U, Dell'Osso L, Carney C. Sleep-related cognitive processes, arousal, and emotion dysregulation in insomnia disorder: the role of insomniaspecific rumination. Sleep Med 2017;30:97-104.

188. Palagini $L$, et al. Daytime rumination as a feature of insomnia disorder: sleep related cognition is not merely a problem of the night. Arch Ital Biol 2015;153:239-47.

189. Lund $H G$, Reider BD, Whiting AB, Prichard JR. Sleep patterns and predictors of disturbed sleep in a large population of college students. J Adolesc Heal. 2010;46:124-32.

190. Takano K, lijima Y, Tanno Y. Repetitive thought and self-reported sleep disturbance. Behav Ther 2012;43:779-89.

191. Thomsen DK, Mehlsen MY, Christensen S, Zachariae R. Rumination - relationship with negative mood and sleep quality. Pers Individ Dif. 2003;34:1293-301.

192. Zoccola PM, Dickerson SS, Lam S. Rumination predicts longer sleep onset latency after an acute psychosocial stressor. Psychosom Med 2009;71:771-5.

193. Lancee J, Eisma MC, van Zanten KB, Topper M. When thinking impairs sleep: trait, daytime and nighttime repetitive thinking in insomnia. Behav Sleep Med 2017; 15:53-69.

194. O'Kearney R, Pech M. General and sleep-specific worry in insomnia. Sleep Biol Rhythms. 2014;12:212-5.

195. Singareddy R, Uhde TW. Nocturnal sleep panic and depression: relationship to subjective sleep in panic disorder. J Affect Disord 2009;112:262-6.

196. Uhde TW, Cortese BM, Vedeniapin A. Anxiety and sleep problems: emerging concepts and theoretical treatment implications. Curr Psychiatry Rep 2009;11:269-76.

197. Reiss S, Peterson RA, Gursky DM, McNally RJ. Anxiety sensitivity, anxiety frequency and the prediction of fearfulness. Behav Res Ther 1986;24:1-8.

198. Hoge EA, et al. The role of anxiety sensitivity in sleep disturbance in panic disorder. J Anxiety Disord 2011;25:536-8.

199. Craske MG, et al. Presleep attributions about arousal during sleep: nocturnal panic. J Abnorm Psychol 2002;111:53-62.

200. Craske MG, Freed S. Expectations about sarousal and nocturnal panic. J Abnorm Psychol 1995;104:567-75.

201. Craske MG, Lang AJ, Tsao JC, Mystkowski JL, Rowe MK. Reactivity to interoceptive cues in nocturnal panic. J Behav Ther Exp Psychiatry. 2001;32:173-90.

202. Tsao JCl, Craske MG. Reactivity to imagery and nocturnal panic attacks. Depress Anxiety. 2003;18:205-13.

203. Timpano KR, Carbonella JY, Bernert RA, Schmidt NB. Obsessive compulsive symptoms and sleep difficulties: exploring the unique relationship between insomnia and obsessions. J Psychiatr Res 2014;57:101-7.

204. Del Río-Casanova L, González A, Páramo M, Van Dijke A, Brenlla J. Emotion regulation strategies in trauma-related disorders: Pathways linking neurobiology and clinical manifestations. Rev Neurosci. 2016; https://doi.org/10.1515/ revneuro-2015-0045.

205. Cohen Kadosh $\mathrm{K}$, et al. Using real-time fMRI to influence effective connectivity in the developing emotion regulation network. Neuroimage. 2016; https://doi.org/ 10.1016/j.neuroimage.2015.09.070

206. Ballesio A, Ottaviani C, Lombardo C. Poor cognitive inhibition predicts rumination about insomnia in a clinical sample. Behav Sleep Med 2018;20:1-10.

207. Tsypes A, Aldao A, Mennin DS. Emotion dysregulation and sleep difficulties in generalized anxiety disorder. J Anxiety Disord 2013;27:197-203.

208. Hovland $A$, et al. Subjective sleep quality in relation to inhibition and heart rate variability in patients with panic disorder. J Affect Disord 2013;150:152-5.

209. Pace-Schott EF, et al. Resting state functional connectivity in primary insomnia, generalized anxiety disorder and controls. Psychiatry Res - Neuroimaging. 2017;265:26-34.

210. Diekelmann S, Born J. Slow-wave sleep takes the leading role in memory reorganization. Nat. Rev. Neurosci. 2010; https://doi.org/10.1038/nrn2762-c2.

211. McDevitt EA, Krishnan GP, Bazhenov M, Mednick SC. Cognitive Neuroscience of Memory Consolidation, Studies in Neuroscience, Psychology and Behavioral Economics. 2017; https://doi.org/10.1007/978-3-319-45066-7_13.

212. Walker MP. The role of sleep in cognition and emotion. Ann N Y Acad Sci 2009;1156:168-97.
213. Rasch B, Born J. About sleep's role in memory. Physiol Rev. 2013; https://doi.org/ 10.1152/physrev.00032.2012.

214. Peever J, Fuller PM. The biology of REM sleep. Curr Biol. 2017; https://doi.org/ 10.1016/j.cub.2017.10.026

215. Colvonen PJ, Straus LD, Acheson D, Gehrman P. A Review of the relationship between emotional learning and memory, sleep, and PTSD. Curr Psychiatry Rep. 2019; https://doi.org/10.1007/s1 1920-019-0987-2.

216. Walker MP, van der Helm E. Overnight Therapy? The Role of Sleep in Emotional Brain Processing. Psychol Bull 2009;135:731-48.

217. Pace-Schott EF, Germain A, Milad MR. Sleep and REM sleep disturbance in the pathophysiology of PTSD: the role of extinction memory. Biol Mood Anxiety Disord. 2015; https://doi.org/10.1186/s13587-015-0018-9.

218. Straus LD, Norman SB, Risbrough VB, Acheson DT, Drummond SPA. REM sleep and safety signal learning in posttraumatic stress disorder: a preliminary study in military veterans. Neurobiol Stres. 2018; https://doi.org/10.1016/j. ynstr.2018.07.001.

219. Jovanovic T, Norrholm SD. Neural mechanisms of impaired fear inhibition in posttraumatic stress disorder. Front Behav Neurosci. 2011; https://doi.org/ 10.3389/fnbeh.2011.00044.

220. Marshall AJ, Acheson DT, Risbrough VB, Straus LD, Drummond, SPA. Fear conditioning, safety learning, and sleep in humans. J Neurosci. 2014; https://doi.org/ 10.1523/jneurosci.0478-14.2014.

221. Pace-Schott EF et al. Effects of post-exposure naps on exposure therapy for social anxiety. Psychiatry Res. 2018; https://doi.org/10.1016/j.psychres.2018.10.015.

222. Pace-Schott EF, Verga PW, Bennett TS, Spencer RMC. Sleep promotes consolidation and generalization of extinction learning in simulated exposure therapy for spider fear. J Psychiatr Res 2012;46:1036-44.

223. Kleim B, et al. Sleep enhances exposure therapy. Psychol Med 2014;44:1511-9.

224. Zalta $A K$, et al. Sleep quality predicts treatment outcome in CBT for social anxiety disorder. Depress Anxiety. 2013;30:1114-20.

225. Nishida M, Pearsall J, Buckner RL, Walker MP. REM sleep, prefrontal theta, and the consolidation of human emotional memory. Cereb Cortex. 2009; https://doi. org/10.1093/cercor/bhn155.

226. Wiesner CD, et al. The effect of selective REM-sleep deprivation on the consolidation and affective evaluation of emotional memories. Neurobiol Learn Mem. 2015; https://doi.org/10.1016/j.nlm.2015.02.008.

227. van Marle H. PTSD as a memory disorder. Eur J Psychotraumatol. 2015; https:// doi.org/10.3402/ejpt.v6.27633.

228. Wagner U, Fischer S, Born J. Changes in emotional responses to aversive pictures across periods rich in slow-wave sleep versus rapid eye movement sleep. Psychosom Med. 2002; https://doi.org/10.1097/01.PSY.0000021940.35402.51.

229. Baran B, Pace-Schott EF, Ericson C, Spencer RMC, Processing of emotional reactivity and emotional memory over sleep. J Neurosci. 2012; https://doi.org/ 10.1523/JNEUROSCI.2532-11.2012.

230. Morgenthaler J, et al. Selective REM-sleep deprivation does not diminish emotional memory consolidation in young healthy subjects. PLoS ONE. 2014;9: e89849.

231. Latchoumane CFV, Ngo HVV, Born J, Shin HS. Thalamic spindles promote memory formation during sleep through triple phase-locking of cortical, thalamic, and hippocampal rhythms. Neuron. 2017;95:424-35.e6.

232. Kleim, B, Wysokowsky, J, Schmid, N, Seifritz, E, Rasch, B. Effects of sleep after experimental trauma on intrusive emotional memories. Sleep. 2016;39:2125-32.

233. Fisher C, Byrne J, Edwards A, Kahn E. A psychophysiological study of nightmares. J Am Psychoanal Assoc 1970;18:747-82.

234. Ross RJ, et al. Rapid eye movement sleep disturbance in posttraumatic stress disorder. Biol Psychiatry. 1994;35:195-202.

235. Kobayashi I, Mellman TA, Altaee D, Howell MK, Lavela J. Sleep and processing of trauma memories. J Trauma Stress. 2016;29:568-71.

236. Levin R, Nielsen TA. Disturbed dreaming, posttraumatic stress disorder, and affect distress: a review and neurocognitive model. Psychol Bull 2007;133:482-528.

237. Rothbaum BO, Mellman TA. Dreams and exposure therapy in PTSD. J Trauma Stress. 2001;14:481-90.

238. Phelps AJ, et al. An ambulatory polysomnography study of the post-traumatic nightmares of post-traumatic stress disorder. Sleep. 2018;41: https://doi.org/ 10.1093/sleep/zsx188

239. Wittmann L, Schredl M, Kramer M. Dreaming in posttraumatic stress disorder: a critical review of phenomenology, psychophysiology and treatment. Psychother Psychosom 2007;76:25-39.

240. Spoormaker VI. Sleep and combat-related post traumatic stress disorder (Springer, 2018).

241. Miller KE, Jamison AL, Gala S, Woodward SH. Two independent predictors of nightmares in posttraumatic stress disorder. J Clin Sleep Med 2018;14:1921-7.

242. Husain AM, Miller PP, Carwile ST. REM sleep behavior disorder: potential relationship to post-traumatic stress disorder. J Clin Neurophysiol 2001;18:148-57. 
243. Naegeli $C$, et al. Locus coeruleus activity mediates hyperresponsiveness in posttraumatic satress disorder. Biol Psychiatry 2018:83:254-62.

244. Nir $Y$, Tononi G. Dreaming and the brain: from phenomenology to neurophysiology. Trends Cogn Sci. 2010;14:88-100.

245. Germain A, et al. A window into the invisible wound of war: functional neuroimaging of REM sleep in returning combat veterans with PTSD. Psychiatry Res. 2013;211:176-9.

246. Germain A, et al. Placebo-controlled comparison of prazosin and cognitivebehavioral treatments for sleep disturbances in US Military Veterans. J Psychosom Res 2012;72:89-96.

247. Raskind MA, et al. Reduction of nightmares and other PTSD symptoms in combat veterans by prazosin: a placebo-controlled study. Am J Psychiatry. 2003;160:371-3.

248. Taylor FB, et al. Prazosin effects on objective sleep measures and clinical symptoms in civilian trauma posttraumatic stress disorder: a placebo-controlled study. Biol Psychiatry. 2008;63:629-32.

249. Raskind MA, et al. Trial of prazosin for post-traumatic stress disorder in military veterans. N Engl J Med 2018;378:507-17.

250. Morgenthaler T, et al. Position paper for the treatment of nightmare disorder in adults: an american academy of sleep medicine position paper. J Clin Sleep Med 2018;14:1041-55.

251. Raskind MA, et al. Higher pretreatment blood pressure is associated with greater posttraumatic stress disorder symptom reduction in soldiers treated with prazosin. Biol Psychiatry. 2016;80:736-42.

252. Richards A, et al. No access an open-label study of doxazosin extended-release for PTSD: findings and recommendations for future research on doxazosin. $J$ lifelong Learn psychiatry. 2018;16:67-73.

253. Aurora $\mathrm{RN}$, et al. Best practice guide for the treatment of nightmare disorder in adults. J Clin Sleep Med. 2010;6:389-401.

254. Krystal $\mathrm{JH}$, et al. It is time to address the crisis in the pharmacotherapy of posttraumatic stress disorder: a consensus statement of the PTSD Psychopharmacology Working Group. Biol Psychiatry. 2017;82:e51-e59.

255. Hertzberg MA, Feldman ME, Beckham JC, Davidson JRT. Trial of trazodone for posttraumatic stress disorder using a multiple baseline group design. J Clin Psychopharmacol. 1996;16:294-8.

256. Villarreal G, et al. Efficacy of quetiapine monotherapy in posttraumatic stress disorder: a randomized, placebo-controlled trial. Am J Psychiatry. 2016;173:1205-12.

257. Kozaric-Kovacic D, Pivac N. Quetiapine treatment in an open trial in combatrelated post-traumatic stress disorder with psychotic features. Int J Neuropsychopharmacol. 2007;10:253-61.

258. De Lecea L. Hypocretins and the neurobiology of sleep-wake mechanisms. Prog Brain Res. 2012;198:15-24.

259. Tao $\mathrm{R}$, et al. Differential effect of orexins (hypocretins) on serotonin release in the dorsal and median raphe nuclei of freely behaving rats. Neuroscience. 2006;141:1101-5.

260. Bonnavion P, De Lecea L. Hypocretins in the control of sleep and wakefulness. Curr Neurol Neurosci Rep. 2010;10:174-9.

261. Grafe LA, Bhatnagar S. Orexins and stress. Front Neuroendocrinol. 2018;51:132-45

262. Carter M, De Lecea L. Hyperarousal and post-traumatic stress disorder: a role for the hypocretin system. In: LeDoux J., Keane T., Shiromani P. (eds) Post-Traumatic Stress Disorder (Humana Press, 2009). https://doi.org/10.1007/978-1-60327-329-9 9.

263. Johnson PL, Molosh A, Fitz SD, Truitt WA, Shekkar A. Orexin, stress, and anxiety/ panic states. Prog Brain Res. 2012;198:133-61.

264. Bonaventure $P$, et al. A selective orexin-1 receptor antagonist attenuates stressinduced hyperarousal without hypnotic effects. J Pharmacol Exp Ther 2015;352:590-601.

265. Möhler H, Fritschy JM, Rudolph U. A new benzodiazepine pharmacology. J Pharmacol Exp Ther 2002;300:2-8.

266. Quadrelli S, Mountford C, Ramadan S. Systematic review of in-vivo neuro magnetic resonance spectroscopy for the assessment of posttraumatic stress disorder. Psychiatry Res. 2018;282:110-25.
267. Starcevic V. The reappraisal of benzodiazepines in the treatment of anxiety and related disorders. Expert Rev Neurother. 2014;14:1275-86.

268. Guina J, et al. Benzodiazepines for PTSD: a systematic review and meta-analysis. J Psychiatr Pract 2015;21:281-303.

269. Pollack MH, et al. Eszopiclone for the treatment of posttraumatic stress disorder and associated insomnia: a randomized, double-blind, placebo-controlled trial. J Clin Psychiatry. 2011;72:892-7.

270. Dunlop BW, et al. Corticotropin-releasing factor receptor 1 antagonism is ineffective for women with posttraumatic stress disorder. Biol Psychiatry. 2017;82:866-74.

271. Davis CJ, Krueger JM. Sleep and cytokines. Sleep Medicine Clinics. 2012;7:517-527.

272. Küffer A, et al. Altered overnight levels of pro-inflammatory cytokines in men and women with posttraumatic stress disorder. Psychoneuroendocrinology. 2019:102:114-20.

273. Babson KA, Sottile J, Morabito D. Cannabis, Cannabinoids, and sleep: a review of the literature. Curr Psychiatry Rep. 2017;19:23.

274. Boden MT, Babson KA, Vujanovic AA, Short NA, Bonn-Miller MO. Posttraumatic stress disorder and cannabis use characteristics among military veterans with cannabis dependence. Am J Addict. 2013;22:277-84.

275. Pietrzak RH, et al. Cannabinoid type 1 receptor availability in the amygdala mediates threat processing in trauma survivors. Neuropsychopharmacology. 2014;39:2519-28.

276. Ney LJ, Matthews A, Bruno R, Felmingham KL. Cannabinoid interventions for PTSD: where to next? Prog Neuro-Psychopharmacol Biol Psychiatry. 2019;93:124-40.

277. Cano G, Mochizuki T, Saper CB. Neural circuitry of stress-induced insomnia in rats. J. Neurosci. 2008;28:10167-84.

278. Mellman TA, David D, Kulick-Bell R, Hebding J, Nolan B. Sleep disturbance and its relationship to psychiatric morbidity after Hurricane Andrew. Am J Psychiatry. 1995:152:1659-63.

279. Mellman TA, Pigeon WR, Nowell PD, Nolan B. Relationships between REM sleep findings and PTSD symptoms during the early aftermath of trauma. J Trauma Stress. 2007;20:893-901.

280. Zuj DV, et al. Impaired fear extinction associated with ptsd increases with hourssince-waking. Depress Anxiety (2016). https://doi.org/10.1002/da.22463.

281. Pace-Schott EF, et al. Extinction of conditioned fear is better learned and recalled in the morning than in the evening. J Psychiatr Res. 2013;47:1776-84.

282. Schuch JB, Genro JP, Bastos CR, Ghisleni G, Tovo-Rodrigues L. The role of CLOCK gene in psychiatric disorders: evidence from human and animal research. Am J Med Genet Part B Neuropsychiatr Genet. 2018;177:181-98.

283. Liu C, Chung M. Genetics and epigenetics of circadian rhythms and their potential roles in neuropsychiatric disorders. Neurosci Bull. 2015;31: 141-59.

284. Mukhopadhyay S, et al. Delayed sleep phase in severe obsessive-compulsive disorder: a systematic case-report survey. CNS Spectr 2008;13:406-13.

285. Turner J, et al. A prospective study of delayed sleep phase syndrome in patients with severe resistant obsessive-compulsive disorder. World Psychiatry. 2007;6:108-11.

286. Coles ME, Schubert JR, Sharkey KM. Delayed bedtimes and obsessivecompulsive symptoms. Behav Sleep Med 2012;10:258-65.

287. Cox RC, Tuck B, Olatunji BO. The role of eveningness in obsessive-compulsive symptoms: cross-sectional and prospective approaches. J Affect Disord 2018;235:448-55.

288. Kaufman J, Charney D. Comorbidity of mood and anxiety disorders. Depress Anxiety. 2000. https://doi.org/10.1002/1520-6394(2000)12:1+<69::AID-DA9>3.0. $\mathrm{CO} ; 2-\mathrm{K}$.

289. Brady KT, Killeen TK, Brewerton T, Lucerini S. Comorbidity of psychiatric disorders and posttraumatic stress disorder. J Clin Psychiatry. 2000;61: 22-32.

290. Dias BG, Banerjee SB, Goodman JV, Ressler KJ. Towards new approaches to disorders of fear and anxiety. Curr Opin Neurobiol. 2013;23:346-52. 